\title{
Semiclassical Yang-Mills Theory I: Instantons
}

\author{
David Groisser $^{1, \star}$ and Thomas H. Parker ${ }^{2, \star \star}$ \\ 1 Department of Mathematics, University of Florida, Gainesville, FL 32611, USA \\ 2 Department of Mathematics, Michigan State University, East Lansing, MI 48824-1027, USA
}

Received December 12, 1989; in revised form July 2, 1990

\begin{abstract}
The partition functions of quantum Yang-Mills theory have an expansion in powers of the coupling constant; the leading order term in this expansion is called the semiclassical approximation. We study the semiclassical approximation for Yang-Mills theory on a compact Riemannian 4-manifold using geometric techniques, and do explicit calculations for the case when the manifold is the 4-sphere. This involves calculating the Riemannian measure and certain functional determinants on the moduli space of self-dual connections. The main result is that the contribution to the semiclassical partition functions coming from the $k=1$ connections on the 4 -sphere is finite and calculable. We also discuss a renormalization procedure in which the radius of the 4-sphere is allowed to tend to infinity.
\end{abstract}

\section{Introduction}

In previous articles ([GP 1, GP 2, and Gr]) the authors have described the Riemannian geometry of the moduli space of self-dual connections on compact 4-manifolds. In this paper we extend those results to address a question of more direct physical interest: the geometry of the semiclassical approximation to the partition functions for Yang-Mills theories on such manifolds. From a geometric perspective these semiclassical approximations arise as follows.

Given a principal $G$-bundle $P$ over a compact oriented Riemannian 4-manifold $(M, g)$, let $\mathscr{A}_{P}$ and $\mathscr{G}_{P}$ be the space of connections and the gauge group of $P$. Let $\mathscr{A}=\mathscr{A}(M)$ be the disjoint union of the $\mathscr{A}_{P}$ over all equivalence classes of bundles $P \rightarrow M$. The quantum expectation of a gauge-invariant function $\Phi: \mathscr{A} \rightarrow \mathbf{R}$ is defined formally as a quotient of two integrals over $\mathscr{A}$ :

$$
\langle\Phi\rangle=\frac{\int_{\mathscr{A}} \Phi(A) e^{-S} d \mathscr{A}}{\int_{\mathscr{A}} e^{-S} d \mathscr{A}}=\frac{Z(\Phi)}{Z(1)} .
$$

\footnotetext{
* Partially supported by N.S.F. grant DMS- 8905211
}

$\star \star$ Partially supported by N.S.F. grant DMS- 8802885 
Here $S(A)=\frac{1}{\xi^{2}} \int_{M}\left|F_{A}\right|^{2}$ (where $F_{A}$ is the curvature 2-form of $A$ and $\xi$ is a parameter) is the Yang-Mills action and $\mathscr{D} \mathscr{A}$ is a measure on $\mathscr{A}$. The main problem of quantum field theory is to make sense of the quantities $Z(\Phi)$ and to compute them explicitly.

Of course, there are formidable problems in defining this measure, and without such a definition the above expression is meaningless. However, if one assumes (as physicists do) that $Z(\Phi)$ is well-defined and that the usual rules of calculus can be applied, then (0.1) can be simplified in two steps. First, one can rewrite $Z(\Phi)$ as an integral over $\mathscr{A} / \mathscr{G}$; this introduces a factor - the "Faddeev-Popov ghost determinant" - into the integrand. Second, the action $S$ depends on the parameter $\xi$ (the coupling constant) and $Z(\Phi)$ has an asymptotic expansion in powers of $\xi$. The leading-order term in this expansion is called the one-loop, or semiclassical, approximation to the expectation. By a "stationary phase" formula this leading term reduces to an integral over the self-dual moduli space $\mathscr{M} \subset \mathscr{A} / \mathscr{G}$. The semiclassical expectation then has the form

$$
\langle\Phi\rangle_{\mathrm{sc}}=\frac{Z(\Phi)}{Z(1)}, \quad \text { where } Z(\Phi)=\int_{\mathscr{M}} \Phi(A) e^{-s} \delta(A) d \mathscr{M},
$$

where $\delta(A)$ is a determinantal factor and $d \mathscr{A}$ is the volume form of the $L^{2}$ Riemannian metric $\mathbf{g}$ on the moduli space.

All of this is described in detail in Sects. 1 and 2. We have paid careful attention to the effect of singularities in $\mathscr{M}$. This effect requires more delicate handling than it has received in the physics literature, where our Theorem 1.1, Proposition 2.1, and certain corollaries are implicitly assumed. In Sects. 3-5 we explicitly calculate the factor $\delta(A) d \mathscr{M}$ for the fundamental example of the $k=1$ moduli space $\mathscr{M}_{1}$ on $S^{4}$. This involves laborious calculations of functional determinants. The basic definitions and our method of calculation are described at the beginning of Sect. 3 . Our main result is that the integrals over $\mathscr{M}_{1}$ in the semiclassical approximation are finite and calculable.

Theorem 1. Let $\mathscr{M}_{1}=\mathscr{M}_{1}\left(S^{4}\right)$ be the moduli space of $k=1$ self-dual connections on the unit 4-sphere. Then for any smooth, bounded, gauge-invariant function $\Phi$ on $\mathscr{M}_{1}$, the semiclassical partition function

$$
\mathbf{Z}_{\mathrm{sc}}(\Phi)=\int_{\mathscr{M}_{1}} \Phi(A) e^{-S} \delta(A) d \mathscr{M}
$$

is finite. Moreover, one has explicit formulas giving the semiclassical measure $\delta v$ $=\delta(A) d \mathscr{M}$ on $\mathscr{M}_{1}\left(S^{4}\right)$.

The formulas for the semiclassical measure are given in Sect. 5. These formulas are complicated and cannot reasonably be integrated in closed form. However, using them it is straightforward to numerically calculate the semiclassical partition functions (0.3).

As an application, we calculate the semiclassical expectations of the instanton number $|k|$ and the scale function $\lambda$. Recall that each connection $A$ has a scale size $\lambda>0$, defined as the radius of the smallest ball containing half the action. This function $\lambda$ is a smooth bounded function on $\mathscr{M}$. In Sect. 6 we show that the semiclassical expectation of $|k|$ and $\lambda$ can be expressed in terms of integrals over $\mathscr{M}_{1}$ (there is no contribution from the moduli spaces of connections with $|k|>1$ ). We carry out the computations in Sect. 7, obtaining

$$
\langle|k|\rangle_{\mathrm{sc}} \approx 0.05958 C_{2} \xi^{-4} e^{-8 \pi^{2} / \xi^{2}}
$$


and

$$
\langle\lambda\rangle_{\mathrm{sc}} \approx 0.03693 C_{2} \xi^{-4} e^{-8 \pi^{2} / \xi^{2}},
$$

where $\xi^{2}$ is a "coupling constant" (denoted $g^{2}$ in the physics literature) and $C_{2}$ is a universal constant (independent of the observable and $\xi$ ) given in Eq. (6.7).

The final section of the paper describes the renormalization of this theory. While the semiclassical partition functions $(0.3)$ have been defined and calculated without introducing a cutoff, the results do depend on two choices of scale: the coupling constant $\xi$, and the radius $R$ of the sphere $S^{4}$. For physical reasons one would like to replace the sphere by $\mathbf{R}^{4}$. To this end, we examine the scaling behavior of the semiclassical partition functions as $R \rightarrow \infty$. We then show how, by choosing $\xi$ to be an appropriate function of $R$, one obtains a finite limit as $R \rightarrow \infty$. The result is that we obtain well-defined and calculable expressions for the semiclassical partition functions and semiclassical expectations on $\mathbf{R}^{4}$. In particular, the renormalized semiclassical expectations of $|k|$ and $\lambda$ are

$$
\langle|k|\rangle_{\mathrm{sc}}^{\mathrm{re}} \approx 0.05958 K_{1}
$$

and

$$
\langle\lambda\rangle_{\mathrm{sc}}^{\mathrm{re}} \approx 0.03693 K_{1}
$$

for a certain constant $K_{1}$.

Actually there is a suppressed length scale in (0.7) and (0.5), the physical unit of length $L$-e.g. $1 \mathrm{~cm}$ or 1 light-year - with respect to which we are calling $S^{4}$ the unit sphere; the physical radius of the sphere is $R L$, not $R$. ( $L$ is effectively the reciprocal of the "mass scale" $\mu$ found in the physics literature; see Sect. 7.) The value of $\langle\lambda\rangle$ given in (0.7) is thus the numerical multiplier of $L$; i.e. one should re-express $(0.7)$ more precisely as

$$
\langle\lambda\rangle_{\mathrm{sc}}^{\mathrm{re}} \approx 0.03693 K_{1} L \text {. }
$$

Similarly, all distances in the theory are given in terms of $L$, the only remaining arbitrary parameter. Thus, while classical instantons on $R^{4}$ have no preferred scale (because of conformal invariance of the self-duality equations), our semiclassical theory has broken the conformal invariance by introducing the fundamental length scale $L$. The importance of $(0.6)$ and (0.8), then, is that in principle $K_{1}$ and $L$ could be determined by measurements of the two observables $\langle|k|\rangle$ and $\langle\lambda\rangle$ (or more generally any two observables of different dimensions.

We note that the above approach - first working on the sphere and then renormalizing to obtain semiclassical expectations on $\mathbf{R}^{4}$ - avoids the infrared divergences which have prevented physicists from completing such calculations (cf. [C], Chap. 7, Sect. 3.6).

The semiclassical measure for instantons has been discussed in numerous physics papers, beginning with 't Hooft's study of the 1-instanton case $[\mathrm{H}]$; a general description of the physicists' approach, as well an extensive list of references, can be found in [O]. The physicists work on $S^{4}$, using the AtiyahDrinfeld-Hitchin-Manin description of instantons [AHDM], for the purpose of regularizing an ill-defined measure over the space of fields on $\mathbf{R}^{4}$. In this process they frequently pass back and forth between $S^{4}$ and $\mathbf{R}^{4}$, attempting to find the "right" flat-space limits of different ingredients of the semiclassical measure, in ways that are not always satisfactorily justified. Our philosophy is to renormalize expectations obtained from $S^{4}$, rather than to renormalize the measure itself. It is 
not obvious whether this approach is equivalent to that of the physicists, although many of the calculations we present can also be found in the physics literature, and our intermediate results, where comparable, agree with what is there. In this paper we have attempted to place the semiclassical approach on a more methodical and rigorous basis than we have been able to find elsewhere. In addition, we have been able to obtain some explicit final answers.

The results of this paper concern only pure gauge fields. Any physically interesting semiclassical theory should also involve fermions. Fermions are incorporated as operators on vector bundles over the space of connections, and the semiclassical partition functions $(0.3)$ are replaced by similar integrals in which one first integrates over the fermions, and then over the moduli space. These issues will be described in part II of this paper.

The physical underpinnings for many of the ideas we use are described in the papers of Coleman [C]. Certain parts of our determinant claculation can also be found in papers of Schwarz [Sc], Romanov and Schwarz [RoSc], and Belavin and Polyakov [BP]. Finally, we thank B. Drachman for help with some of the numerical analysis done in Sect. 7.

\section{The Moduli Space}

In this section we briefly review the standard global-analytic description of the selfdual moduli space. Details can be found in Sect. 1 of [GP 1], and there are numerous general references ([AHS, FU, and L]). Our aim here is simply to fix notation, introduce some basic constructions that will be used later, and recall the basic theorem concerning the $L^{2}$ geometry of the $k=1$ moduli space over $S^{4}$.

Let $(M, g)$ be a compact, oriented, Riemannian 4-manifold and $P \rightarrow M$ a principal bundle whose structure group is a compact simple Lie group $G$ with Lie algebra $\mathfrak{g}$, and whose instanton number is $k \in \mathbf{Z}$. We can then consider the spaces $\Omega^{p}(\operatorname{Ad}(P))$ of $p$-forms with values in the associated adjoint bundle $\operatorname{Ad}(P)=P \times{ }_{\text {Ad }} g$. There is a one-parameter family of invariant inner products on the Lie algebra $g$ given by

$$
(X, Y)_{\xi}=-\frac{1}{4 \xi^{2}} K(X, Y)
$$

where $K$ is the Killing form. [The factor $1 / 4$ is chosen so that when $G=S U(2)$ and $\xi=1,(\cdot, \cdot)_{\xi}$ is (minus) the trace form of the standard representation. This normalization makes it easier to compare our formulas with others in the literature.] These inner products on $g$ induce inner products on the $\Lambda^{p}\left(T^{*} M\right) \otimes \operatorname{Ad}(P)$, and hence $L^{2}$ inner products on $\Omega^{p}(\operatorname{Ad}(P))$ :

$$
\langle\phi, \psi\rangle_{\xi}=\int_{M}(\phi, \psi)_{\xi} d M,
$$

where $d M$ is the volume form on $M$ determined by the metric $g$. The "coupling constant" $\xi$ thus labels a 1-parameter family of inner products. (Physicists write $g$ for the coupling constant, but for us $g$ is a metric.) While in classical Yang-Mills theory the only role of $\xi$ is to trivially rescale some norms, in the quantum theory $\xi$ becomes a fundamental parameter. In fact, as we will see in the next section, varying $\xi$ gives rise to a 1-parameter family of quantum Yang-Mills theories. 
A connection $A$ on $P$ determines a covariant derivative $\nabla^{A}$, an exterior covariant derivative $d_{A}$, and a fundamental elliptic sequence

$$
0 \rightarrow \Omega^{0}(\operatorname{Ad}(P)) \stackrel{d_{A}}{\longrightarrow} \Omega^{1}(\operatorname{Ad}(P)) \stackrel{d_{\bar{A}}}{\longrightarrow} \Omega_{-}^{2}(\operatorname{Ad}(P)) \rightarrow 0 .
$$

[Here, and in other notations below, the minus signs denote composition with the pointwise projection $p_{-}=\frac{1}{2}(1-*)$ onto the subspace $\Omega_{-}^{2} \subset \Omega^{2}$ of anti-self-dual 2-forms.] Using the metric, we obtain formal adjoints $d_{A}^{*}$ and $\left(d_{A}^{-}\right)^{*}$ to $d_{A}$ and $d_{A}^{-}$, and hence Laplacians

$$
\Delta_{A}^{0}=d_{A}^{*} d_{A}, \quad \Delta_{A}^{1}=d_{A} d_{A}^{*}+2\left(d_{A}^{-}\right)^{*} d_{A}^{-}, \quad \Delta_{A}^{-}=2 d_{A}^{-}\left(d_{A}^{-}\right)^{*}
$$

on the spaces in the above complex. These are elliptic operators, so each space $\Omega^{q}(\operatorname{Ad} P)$ decomposes into eigenspaces of the Laplacian. In particular, there is an $L^{2}$-orthogonal decomposition $\Omega^{q}(\operatorname{Ad}(P))=K^{q} \oplus B^{q}$, where $K^{q}=\operatorname{ker}\left(\Delta_{A}^{q}\right)$, and a Green operator $G_{A}^{q}: B^{q} \rightarrow B^{q}$ that inverts the Laplacian on $B^{q}$.

Remark. Using $d_{A}^{*}=-* d_{A^{*}}$ one finds that

$$
2 d_{A}^{-}\left(d_{A}^{-}\right)^{*}=d_{A} d_{A}^{*}+d_{A}^{*} d_{A} .
$$

Thus $\Delta_{A}^{-}$is simply the restriction to $\Omega_{-}^{2}(\operatorname{Ad} P)$ of the ordinary covariant Laplacian on $\Omega^{2}(\operatorname{Ad} P)$ (this is why we choose to have a factor of 2 in our definition of $\Delta_{A}^{-}$.) The factor of 2 in the definition of $\Delta_{A}^{1}$ is inserted so that the Laplacians $\Delta_{A}^{1}$ and $\Delta_{A}^{-}$ will intertwine with $d_{A}^{-}$and its adjoint.

The curvature of the connection $A$ is the AdP-valued 2-form $F_{A}=d_{A} \circ d_{A}$. Projecting onto the subspaces $\Omega_{ \pm}^{2}(\operatorname{Ad} P)$ gives a decomposition $F_{A}=F_{A}^{+}+F_{A}^{-}$of $F_{A}$ into self-dual and anti-self-dual parts. In terms of these and the inner product (1.1) the instanton number is

$$
k=\frac{\xi^{2}}{8 \pi^{2}} \int_{M}\left(\left|F_{A}^{+}\right|_{\xi}^{2}-\left|F_{A}^{-}\right|_{\xi}^{2}\right) d M
$$

and the Yang-Mills action is

$$
S_{\xi}(A)=\int_{M}\left(\left|F_{A}^{+}\right|_{\xi}^{2}+\left|F_{A}^{-}\right|_{\xi}^{2}\right) d M=\frac{1}{\xi^{2}} S_{1}(A) .
$$

Note that the $S_{\xi}$ form a 1-parameter family of actions. Now observe that

$$
S_{1}(A)=8 \pi^{2} k+2 \int\left|F_{A}^{-}\right|_{1}^{2}=-8 \pi^{2} k+2 \int\left|F_{A}^{+}\right|_{1}^{2} .
$$

Hence when $k \geqq 0, S_{\xi}(A)$ achieves its absolute minimum whenever the connection $A$ is self-dual; i.e. whenever $F_{A}^{-}=0$. Likewise, when $k \leqq 0$ the absolute minimum is achieved when $F_{A}^{+}=0$; i.e. $A$ is anti-self-dual. In either case when $G=S U(2)$ he value of the minimum value of $S_{\xi}$ is $8 \pi^{2} \xi^{-2}|k|$. For most of this paper we will explicitly discuss only the self-dual case; the anti-self-dual case is entirely analogous.

To treat the infinite-dimensional spaces derived from $\mathscr{A}$ as manifolds requires some global analysis. Fix an $s>1$ and let $\mathscr{A}$ denote the affine Hilbert space obtained by completing the space of all smooth connections on $P$ in the Sobolev $L_{s}^{2}$-norm. Similarly, let $\mathscr{G}$ be the $L_{s+1}^{2}$-completion of the gauge group (the group of automorphisms of $P$ ). Then $\mathscr{G}$ is a Hilbert Lie group that acts smoothly on $\mathscr{A} ;$ let $\mathscr{B}=\mathscr{A} / \mathscr{G}$ be the orbit space. The center $\mathscr{Z}$ of $\mathscr{G}$ is a finite group (isomorphic to the center of $G$ ) that acts trivially on $\mathscr{A}$. The quotient $\widetilde{\mathscr{G}}=\mathscr{G} / \mathscr{Z}$ acts freely on the open 
dense set $\mathscr{A}^{*}$ of irreducible connections, and $\mathscr{B}^{*}=\mathscr{A}^{*} / \widetilde{\mathscr{G}}=\mathscr{A}^{*} / \mathscr{G}$ is a Hilbert manifold.

Moreover, these spaces $\mathscr{A}, \mathscr{G}$, and $\mathscr{B}^{*}$ are Riemannian manifolds - each has a strong $L_{s}^{2}$ metric and a weak $L^{2}$ metric, obtained as follows. First, at each $A \in \mathscr{A}$ we can identify $T_{A} \mathscr{A}=L_{s}^{2}\left(T^{*} M \otimes \operatorname{Ad} P\right)$; the $L_{s}^{2}$ and $L^{2}$ metrics on $T^{*} M \otimes \operatorname{Ad} P$ thus induce translation-invariant Riemannian metrics on $\mathscr{A}$. Similarly $T_{g} \mathscr{G}$ $=L_{s+1}^{2}(\operatorname{Ad} P)$ at each $g \in \mathscr{G}$, so we obtain $L_{s+1}^{2}$ and $L^{2}$ metrics on $\mathscr{G}$. The tangent

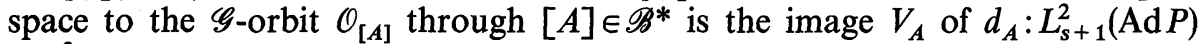
$\left.\rightarrow L_{s}^{2}\left(T^{*} M \otimes \mathrm{Ad} P\right)\right)$ - the extension of the first map in (1.2). This is the "vertical subspace" for the projection $\mathscr{A}^{*} \rightarrow \mathscr{B}^{*}$. Its $L^{2}$-orthogonal complement gives a horizontal space $H_{A}=\operatorname{ker} d_{A}^{*} \subset T_{A} \mathscr{A}=L_{s}^{2}\left(T^{*} M \otimes A d P\right)$, so we can make the identification

$$
T_{[A]} \mathscr{B}^{*}=H_{A} .
$$

The metrics $\mathbf{g}_{s}$ and $\mathbf{g}$ on $\mathscr{B}^{*}$ are then given by restricting the $L_{s}^{2}$ and $L^{2}$ metrics on $\mathscr{A}$ to this horizontal subspace $H_{A}$. Specifically, using the notation $\square_{A}^{p}=1+\Delta_{A}^{p}$, the norm of $\left.X \in T_{[A]} \mathscr{B}^{*}=H_{A} \subset L_{s}^{2}\left(T^{*} M \otimes \operatorname{Ad} P\right)\right)$ is given by

$$
\left(\mathbf{g}_{s}\right)_{A}(X, X)=\langle X, X\rangle_{L_{s}^{2}}=\left\langle X,\left(\square_{A}^{1}\right)^{s} X\right\rangle_{L^{2}} .
$$

With these definitions $\mathscr{A} \rightarrow \mathscr{B}^{*}$ is a Riemannian submersion with respect to both these metrics.

Remarks. (1) It is the $L^{2}$ metric that arises naturally in physics, but the "regularized" metric $L_{s}^{2}$ must be introduced to obtain this global description and to prove many technical analytic facts (cf. [GP 2], Sect. 1).

(2) Note that the metrics on $\mathscr{A}, \mathscr{B}^{*}$, and $\mathscr{G}$ depend on $\xi$, but $\Delta_{A}^{p}$ does not.

The moduli space $\mathscr{M}$ of self-dual connections is the subset of $\mathscr{B}$ consisting of the gauge orbits $[A]$ of connections with $F_{A}^{-}=0$. In general, $\mathscr{M}$ is finite-dimensional and has singularities at those orbits $[A]$ where either $\operatorname{ker} \Delta_{A}^{0} \neq\{0\}$ (i.e. $A$ is a reducible connection) or $\operatorname{ker} \Delta_{A}^{-} \neq\{0\}$. For a generic metric the second type of singularity will not occur (see [FU]), and we can eliminate the first type of singularity by restricting attention to $\mathscr{M}^{*}=\mathscr{M} \cap \mathscr{B}^{*}$, which is then a smooth manifold. Thus for a generic metric we have a diagram

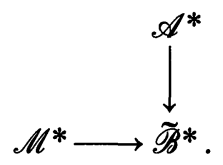

Restricting the $L^{2}$ metric on $\mathscr{B}^{*}$ then makes $\mathscr{M}^{*}$ a finite-dimensional Riemannian manifold. (In fact, the restrictions of the $L^{2}$ and $L_{s}^{2}$ metrics are equal on $\mathscr{M}^{*}$.) We will denote this metric on $\mathscr{M}^{*}$ by $\mathrm{g}$ and will often use the letter $A$ to mean both a connection and the corresponding point $[A] \in \mathscr{M}$.

This description can be refined by introducing a space $\tilde{\mathscr{M}}$ that resolves the "type-one" singularities of $\mathscr{M}$. Fix a basepoint $x_{0} \in M$, let $\mathscr{G}^{0}$ be the group of gauge transformations that are the identity at $x_{0}$, and let $\mathscr{G}_{s+1}^{0}$ be its completion in the Sobolev $(s+1)$-norm. It was shown in [GP 2, Sect. 1] that $\mathscr{G}_{s+1}^{0}$ acts smoothly on $\mathscr{A}_{s}$, that $\tilde{\mathscr{B}}=\mathscr{A}_{s} / \mathscr{G}_{s+1}^{0}$ is a manifold, and that $\mathscr{A}_{s} \rightarrow \mathscr{B}$ is a smooth Riemannian submersion with respect to the $L_{s}^{2}$ Riemannian metrics. Restricting to the self-dual connections $\mathscr{S} \mathscr{D} \subset \mathscr{A}$ gives a fibration $\mathscr{S} \mathscr{D} \rightarrow \tilde{M}=\mathscr{S} \mathscr{D} / \mathscr{G}^{0}$. The Sobolev metrics on $\mathscr{A}$ induce a family of metrics $\left\{\mathbf{g}_{s} \mid s>1\right\}$ on $\tilde{\mathscr{M}}$ as described in Theorem 1.2 of [GP 2]. The corresponding theorem for the $L^{2}$ metric is the following. 
Theorem 1.1. Suppose that $(M, g)$ is a Riemannian manifold with constant positive scalar curvature. Then there exists a smooth Riemannian metric $\tilde{\mathbf{g}}$ on $\tilde{\mathscr{M}}$ such that $\mathscr{S} \mathscr{D} \rightarrow \tilde{\mathscr{M}}=\mathscr{S} \mathscr{D} / \mathscr{G}_{0}$ is a Riemannian submersion with respect to the $L^{2}$ metric on $\mathscr{S} \mathscr{D}$ and the metric $\tilde{\mathbf{g}}$ on $\tilde{\mathscr{M}}$. Furthermore, $\mathscr{G}_{s+1} / \mathscr{G}_{s+1}^{0} \cong G / Z$ acts isometrically on $(\tilde{\mathscr{M}}, \tilde{\mathbf{g}})$. This action is free and effective on the irreducible connections $\tilde{M}^{*} \subset \tilde{\mathscr{M}}$, and the orbitspace projection $\left(\tilde{\mathscr{M}}^{*}, \tilde{\mathbf{g}}\right) \rightarrow\left(\mathscr{M}^{*}, \mathbf{g}\right)$ is a Riemannian submersion.

The metric $\tilde{\mathbf{g}}$ on $\tilde{\mathscr{M}}$ in this theorem is obtained by analytically continuing the metrics $\mathbf{g}_{s}$ to $s=0$. This requires a minor modification in the definition of $\mathbf{g}_{s}$ (replacing the operator $\square=\Delta+1$ above by $\Delta+R / 12$ ). The proof is rather involved and will be given in a separate paper.

The most basic self-dual moduli space is the space $\mathscr{M}_{1}=\mathscr{M}_{1}\left(S^{4}, g_{0}\right)$ of $k=1$ $S U(2)$ instantons on the standard unit sphere $S^{4}$. In this case $\mathscr{M}_{1}=\mathscr{M}_{1}^{*}$ is diffeomorphic to $\mathbf{R}^{5}$ (see [AHS]). In [GP 1] the authors defined a specific diffeomorphism

$$
\bar{Q}: \mathbf{R}^{5} \rightarrow \mathscr{M}_{1}
$$

and calculated the metric on $\mathscr{M}_{1}$ in this coordinate system (see also [DMM]). The result was

$$
\bar{Q}^{*} \mathbf{g}=\psi^{2}(\varrho) \sum\left(d x^{i}\right)^{2},
$$

where $\varrho=|x|$ and where $\psi$ is a rather complicated function. This metric has the following geometric properties.

Theorem 1.2. Let $\mathscr{M}_{1}$ be the moduli space $\mathscr{M}_{1}\left(S^{4}, g_{0}\right)$ with its $L^{2}$ Riemannian metric Then its metric space completion $\bar{M}_{1}$

(1) is conformally flat and rotationally symmetric,

(2) has finite radius and volume,

(3) has boundary $\partial \mathscr{M}_{1}$ that is totally geodesic and is isometric to $S^{4}$ with the metric $4 \pi^{2} \xi^{-2} g_{0}$.

The moduli space $\mathscr{M}_{0}\left(S^{4}, g_{0}\right)$ is a single point - the trivial flat connection $\theta$. For $k \neq 0$, the antipodal map on $S^{4}$ induces a pullback map $\mathscr{A}_{k} \rightarrow \mathscr{A}_{-k}$ that preserves the $L^{2}$ metric, and hence gives an isometry between $\mathscr{M}_{k}\left(S^{4}, g_{0}\right)$ and $\mathscr{M}_{-k}\left(S^{4}, g_{0}\right)$. All the singularities in these moduli spaces occur at reducible connections. In fact the following lemma shows that there are no "type-two" singularities in a neighborhood of $\mathscr{M} \subset \mathscr{B}$.

Lemma 1.3. There exists an $\varepsilon>0$ such that any connection $A$ on $\left(S^{4}, g_{0}\right)$ with $\operatorname{ker} \Delta_{\boldsymbol{A}}^{-} \neq\{0\}$ and with instanton number $k$ has

$$
S_{1}(A) \geqq 8 \pi^{2}|k|+\varepsilon .
$$

Proof. Suppose $k \geqq 0$ and that $\phi \in \operatorname{ker} \Delta_{A}^{-}$. The Weitzenböck formula for $\Delta_{A}^{-}$on $S^{4}$ ([FU] Eq. 6.26) shows that $\nabla_{A}^{*} \nabla_{A} \phi+4 \phi+\left[F_{A}^{-}, \phi\right]=0$. [Here

$$
\nabla_{A}: \Gamma\left(\operatorname{Ad} P \otimes \Lambda^{2} T^{*} S^{4}\right) \rightarrow \Gamma\left(\operatorname{Ad} P \otimes \Lambda^{2} T^{*} S^{4} \otimes T^{*} S^{4}\right)
$$

is the tensor product of $A$ with the Levi-Civita connection.] Taking the inner product with $\phi$, integrating by parts, using the pointwise inequality $|d| \phi \| \leqq|\nabla \phi|$, and applying Hölder's inequality gives

$$
\||\phi|\|_{L_{1}^{2}}^{2}=\int\|d|\phi|\|^{2}+|\phi|^{2} \leqq \int_{M}|\phi|^{2}\left|F_{A}^{-}\right| \leqq\left\|F_{A}^{-}\right\|_{L^{2}}\||\phi|\|_{L^{2}}^{2} .
$$


Hence $\left\|F_{A}^{-}\right\|_{L^{2}}^{2}=\frac{1}{2} S_{1}(A)-4 \pi^{2} k \geqq c^{2}$, where $c$ is the constant of the Sobolev embedding $L^{4} \hookrightarrow L_{1}^{2}$ on $S^{4}$. The case $k<0$ is similar.

The remaining singularities in $\mathscr{M}_{k}$ occur at the reducible connections. However, the following lemma (which is needed in the next section) shows that the reducible connections are a small (lower-dimensional) subset of $\widetilde{\mathscr{M}}$. This lemma applies to any $(M, g)$ provided $\tilde{\mathscr{M}}_{k}$ contains no type-two singularities.

Lemma 1.4. For $k \neq 0$ the irreducible connections $[A] \in \tilde{\mathscr{M}}_{k}$ form an open dense set whose complement has codimension at least two.

Proof. By Theorem 1.2 of [GP 2] the action of the gauge group on $\tilde{\mathscr{M}}_{k}$ factors through a smooth action of the compact group $G / Z$. The principal orbits of this action - those of maximal dimension, in this case $\operatorname{dim}(G)$ - include the orbits of all irreducible connections. In fact these are the only principal orbits, since the proof of Theorem $3.1 \mathrm{in}$ [FU] shows that the stabilizer of each reducible connection $A$ is a Lie group of strictly positive dimension. A theorem of Montgomery, Samelson, and Zippin ([MSZ]) on actions by compact connected Lie groups then implies that the set of principal orbits is as stated above.

Each instanton $[A] \in \mathscr{M}_{1}$ has a center point $p \in S^{4}$ and a scale $\lambda$, defined as the radius of the smallest ball around $p$ containing half the action, i.e.

$$
4 \pi^{2} \xi^{-2}=\int_{B_{\lambda}(p)}\left|F_{A}\right|_{\xi}^{2} .
$$

The radial geodesics in $\mathscr{M}_{1}$ are families of connections with a fixed center $p$; these approach the boundary of $\mathscr{M}$ as $\lambda \rightarrow 0$, and $\partial \mathscr{M}_{1}$ consists of formal connections whose curvature is supported at a single point $p \in S^{4}$.

Altogether, this gives a complete description of one component of the configuration space of classical Yang-Mills fields on $S^{4}$ - the component $\mathscr{M}_{1}$ of self-dual $k=1$ fields. (The $k=-1$ component $\mathscr{M}_{-1}$ is isometric to $\mathscr{M}_{1}$.) We will next see how further geometric aspects of the embedding $\mathscr{M} \subset \mathscr{B}$ come into play in the quantum theory.

\section{Euclidean Quantum Field Theory}

Quantum Yang-Mills theory on a compact Riemannian manifold is best approached using path integrals. In this section we briefly review this path integral formulation, casting it as much as possible in the geometric framework described in Sect. 1. We then examine the semiclassical, or "one-loop", approximations to the path integrals and show how they reduce to integrals over finite-dimensional spaces. There is as yet no rigorous mathematical treatment of path integrals of this type. Thus the arguments in this section are of necessity made in the spirit of physics - heuristic, but compelling.

In physics, classical Yang-Mills theory is a field theory on a Lorentzian spacetime 4-manifold $M$. The corresponding quantum theory is constructed by considering the space $\mathscr{A}$ of classical fields (connections) on $M$, and then defining the quantum expectation values using path integrals. The "Euclidean" quantum field theory that we will be studying in this paper is completely analogous, but the spacetime manifold is replaced by a Riemannian manifold $M$, which we will take to be compact. This Euclidean theory is relevant to tunneling amplitudes in the quantum theory (cf. [C], Chap. 7, Sect. 2), and is also of interest in its own right. 
We start with a compact oriented Riemannian 4-manifold $(M, g)$ and a compact Lie group $G$. For each principal $G$-bundle $P$ over $M$ we have the setup described in the previous section: a space $\mathscr{A}_{P}$ of connections on $P$, a gauge group $\mathscr{G}_{P}$, and an orbit space $\mathscr{B}_{P}$. Let $\mathscr{A}=\mathscr{A}(M)$ be the disjoint union of the $\mathscr{A}_{P}$ over all isomorphism classes of principal $G$-bundles over $M$. An observable in the quantum field theory is a gauge-invariant function on $\mathscr{A}$, or equivalently, a function

$$
\Phi: \mathscr{B} \rightarrow \mathbf{R} \text {. }
$$

Examples

(1) Given $A$, let $F_{A}=$ curvature of $A$. For any $p>0$ and $x \in M$,

are observables.

$$
\Phi(A)=\int_{M}\left|F_{A}\right|^{p} \quad \text { and } \quad \Phi(A)=\left|F_{A}(x)\right|^{p}
$$

(2) Fix a loop $\gamma: S^{1} \rightarrow M$ and choose a representation $\varrho$ of $G$. For each $A$, let $P_{\gamma}^{A}$ denote parallel transport (or holonomy) around $\gamma$ with respect to the connection $A$. The "Wilson loop functional" associated to $\gamma$ and $\varrho$ is

$$
\Phi(A)=\operatorname{tr}\left(\varrho\left(P_{\gamma}^{A}\right)\right) .
$$

This is independent of the choice of basepoint on $\gamma$ and is gauge-invariant.

(3) The instanton number $k$ and its absolute value $|k|$ are observables.

(4) Every connection $A$ with $k=1$ has a scale size $\lambda(A)$ defined by (1.9). A simple way of extending $\lambda$ to a continuous function on $\mathscr{A}$ is to define $\lambda(A)$, for $A \in \mathscr{A}_{k}$, as the radius of the smallest ball containing half the minimum action for configurations in $\mathscr{A}_{k}$; i.e.

$$
\lambda(A)=\inf _{r>0}\left\{\text { for some } p \in M, \int_{B_{r}(p)}\left|F_{A}\right|_{\xi}^{2}=4 \pi^{2} \xi^{-2}|k|\right\} .
$$

Then $\lambda \equiv 0$ on $\mathscr{A}_{0}$, and for connections that resemble $k>1$ widely separated instantons, $\lambda$ will be a large number indicating their separation.

Additional observables can be constructed using fermions; in particular given $k>0$ one can obtain operators that vanish in $\mathscr{A}_{l}$ for $|l| \leqq k$. These will be discussed in part II of this paper.

Quantum field theory is a systematic way of computing the average values, or expectations, of such functions. The expectation is defined by the usual formula

$$
\langle\Phi\rangle=\frac{\int_{\mathscr{A}} \Phi(A) \mathscr{D} \mathscr{A}}{\int_{\mathscr{A}} \mathscr{D} \mathscr{A}} .
$$

Here $\mathscr{D} \mathscr{A}$ is a measure on the (infinite-dimensional) space $\mathscr{A}$. The particular choice of measure is determined by physics. Quantum mechanics leads us to think of the field as a random variable, distributed according to a measure concentrated around the solutions of the underlying classical field equations. The measure $\mathscr{D} \mathscr{A}$ describes the strength and nature of these fluctuations around the classical YangMills solutions.

On a compact Riemannian manifold these classical solutions are the self-dual instantons, which are the minima of the Yang-Mills action (1.6). Physicists take the measure on $\mathscr{A}$ to be

$$
\mathscr{D} \mathscr{A}=e^{-S_{\xi}(A)} d \mathscr{A},
$$


where $S_{\xi}(A)$ is the Yang-Mills action (1.6) and $d \mathscr{A}$ is a putative "translationinvariant measure on $\mathscr{A} "$, which can be thought of as the measure associated with the $L^{2}$ Riemannian metric on $\mathscr{A}$. The expectation then has the form commonly found in the physics literature:

$$
\langle\Phi\rangle=\frac{Z(\Phi)}{Z(1)}, \text { where } Z(\Phi)=\int_{\mathscr{A}} \Phi e^{-S_{\xi}} d \mathscr{A} .
$$

The function $Z$ is called the partition function of the quantum field theory. Note that $Z$, and therefore $\langle\Phi\rangle$, depends on $\xi$. Therefore we obtain a 1-parameter family of quantum field theories. (The $L^{2}$ metric on $\mathscr{A}$ also depends on $\xi$, but the dependence of $d \mathscr{A}$ on $\xi$ formally cancels in the quotient $\langle\Phi\rangle$; hence we can assume that $d \mathscr{A}$ is independent of $\xi$.)

We next simplify this formula in two steps. In doing this we will adopt the physicists' pragmatic approach, ignoring the problems of defining the measure, and evaluating the integrals $Z(\Phi)$ by analogy with the finite-dimensional case. To start, we will also assume that $\mathscr{B}$ and $\mathscr{M}$ are smooth manifolds.

Step 1. Rewriting the expectation as an integral over $\mathscr{B}$.

Recall that the $L^{2}$ Riemannian metric on $\mathscr{A}$ descends to give a Riemannian metric on $\mathscr{B}$ in such a way as to make $\mathscr{A} \rightarrow \mathscr{B}$ a Riemannian submersion. Suppose, for the moment, that this fibration were finite-dimensional with compact fibers. Then for any gauge-invariant function $\Phi$ on $\mathscr{A}$ we would have

$$
\int_{\mathscr{A}} \Phi d \mathscr{A}=\int_{\mathscr{B}} \Phi \operatorname{Vol}\left(\mathcal{O}_{\mathscr{A}}\right) d \mathscr{B}=\operatorname{Vol}(\mathscr{G}) \int_{\mathscr{B}} \Phi \sqrt{\operatorname{det}\left(\left(i_{A}\right)^{*}\left(i_{A}\right)_{*}\right)} d \mathscr{B},
$$

where $\operatorname{Vol}\left(\mathcal{O}_{\mathscr{A}}\right)$ is the volume of the gauge orbit through $A \in \mathscr{A}, \operatorname{Vol}(\mathscr{G})$ is the volume of the gauge group, $d \mathscr{B}$ is the volume form of the Riemannian metric on $\mathscr{B}$, and $\left(i_{A}\right)_{*}$ is the differential of the inclusion $i_{A}: \mathscr{G} \rightarrow \mathcal{O}_{A}$ of the gauge group into the orbit through $A$. Applying this formula in our infinite-dimensional situation, we have $\left(i_{A}\right)_{*}=d_{A}$, the first operator in the fundamental elliptic sequence (1.2), so

$$
\sqrt{\operatorname{det}\left(\left(i_{A}\right)^{*}\left(i_{A}\right)_{*}\right)}=\sqrt{\operatorname{det}\left(d_{A}^{*} d_{A}\right)}=\sqrt{\operatorname{det} \Delta_{A}^{0}} .
$$

The factor of $\operatorname{Vol}(\mathscr{G})$ then cancels in the ratio $\langle\Phi\rangle$, so we can rewrite the expectation as an integral over $\mathscr{B}$ :

$$
\langle\Phi\rangle=\frac{Z(\Phi)}{Z(1)}, \quad \text { where } \quad Z(\Phi)=\int_{\mathscr{B}} \Phi e^{-S_{\xi}} \sqrt{\operatorname{det} \Delta^{0}} d \mathscr{B} .
$$

Step 2. The Semiclassical Approximation.

Recall that the Yang-Mills action (1.6) depends on the coupling constant $\xi$. As $\xi \rightarrow 0$ the above integral has an asymptotic expansion in powers of $\xi$; the leadingorder term gives the semiclassical approximation to the expectation. In the finitedimensional case the relevant asymptotic formula is the following. Suppose that $S$ and $\Phi$ are smooth functions on a compact Riemannian manifold $\mathscr{B}$, that the set $\mathscr{M} \subset \mathscr{B}$ of minima of $S$ is a non-degenerate submanifold, and that $\Phi>0$ on $\mathscr{M}$. Then

$$
\int_{\mathscr{B}} \Phi e^{-S / \xi^{2}} d \mathscr{B}=e^{-\bar{S} / \xi^{2}}\left\{\int_{\mathscr{M}} \Phi \operatorname{det}\left(\frac{H_{S}}{2 \pi \xi^{2}}\right)^{-1 / 2} d \mathscr{M}\right\}\left(1+O\left(\xi^{2}\right)\right),
$$

where $\bar{S}$ is the value of $S$ on the manifold $\mathscr{M}$ of minima, $H_{S}$ is the Hessian of $S$ on the normal bundle of $\mathscr{M}$, and $d \mathscr{M}$ and $d \mathscr{B}$ are the measures induced by the Riemannian 
metric on $\mathscr{B}$. In fact, this formula holds for any $\Phi \in C^{1}(\mathscr{B})$ for which the right-hand integral is non-zero. We will also make use of a cruder asymptotic formula: for any decomposition $\mathscr{B}=\mathscr{B}^{-} \cup \mathscr{B}^{+}$with $S<\varepsilon$ on $\mathscr{B}_{-}$and $S \geqq \varepsilon$ on $\mathscr{B}_{+}$we have

$$
\int_{\mathscr{B}} \Phi e^{-S / \xi^{2}}=\int_{\mathscr{B}_{-}} \Phi e^{-S / \xi^{2}}+e^{-\varepsilon / \xi^{2}}\left(\int_{\mathscr{B}_{+}} \Phi e^{-(S-\varepsilon) / \xi^{2}}\right)=\left(\int_{\mathscr{B}_{-}} \Phi e^{-S / \xi^{2}}\right)\left(1+O\left(\xi^{N}\right)\right)
$$

for any $N$. Again, this holds for any $\Phi$ for which the right-hand integral does not vanish.

By definition, the semiclassical approximation is what is obtained by applying these formulas to the infinite-dimensional integral $Z(\Phi)$ in (2.2) and retaining only the leading-order term in $\xi$. For this we take $S$ to be the normalized Yang-Mills action $S_{1}$ and write $\mathscr{B}=\bigcup \mathscr{B}_{k}$, where $\mathscr{B}_{k}$ is the component of $\mathscr{A} / \mathscr{G}$ with instanton number $k$. The minimum of $S_{1}$ on $\mathscr{B}_{k}$ is $\bar{S}=8 \pi^{2}|k|$. By (2.4)

$$
Z(\Phi)=\left(\int_{\mathscr{B}_{0}} \Phi e^{-S_{\xi}} \sqrt{\operatorname{det} \Delta^{0}} d \mathscr{B}\right)\left(1+O\left(\xi^{N}\right)\right) \quad \forall N
$$

unless this integral vanishes. If it does vanish, then

$$
Z(\Phi)=\left(\int_{\mathscr{B}-1} \Phi \mathscr{B}_{1} e^{-S_{\xi}} \sqrt{\operatorname{det} \Delta^{0}} d \mathscr{B}\right)\left(1+O\left(\xi^{N}\right)\right) \quad \forall N
$$

unless this also vanishes, etc. We can now apply (2.3) to these integrals, noting that the submanifold of minima of $S_{1}$ on $\mathscr{B}_{k}$ is the moduli space $\mathscr{M}_{k}$ and that for $k>0$ the restriction to the normal bundle of the Hessian of $S_{1}$ at $A \in \mathscr{M}_{k}$ is

$$
H_{S_{1}}=\left.2\left(d_{A}^{-}\right) * d_{A}^{-}\right|_{N}
$$

where

$$
N=\operatorname{image}\left(d_{A}^{-}\right)^{*}
$$

[see (1.2)]. Note that $H_{S_{1}}$ is strictly positive and that the operators $d_{A}^{-}$and $\left(d_{A}^{-}\right)^{*}$ intertwine it with the Laplacian $2 d_{A}^{-}\left(d_{A}^{-}\right)^{*}=\Delta_{A}^{-}$. This Laplacian therefore has the same spectrum as $H_{S_{1}}$, and hence the same determinant [cf. (3.11)]. Thus for any observable $\Phi$ that vanishes on $\mathscr{B}_{l}$ for $|l|<|k|$ we arrive at the formula

$$
\langle\Phi\rangle_{\mathrm{sc}}=\frac{Z_{k}(\Phi)+Z_{-k}(\Phi)}{Z_{0}(1)}
$$

provided $Z_{k}(\Phi)+Z_{-k}(\Phi) \neq 0$. Here

where

$$
Z_{k}(\Phi)=e^{-8 \pi^{2}|k| / \xi^{2}} \int_{\mathscr{M}_{k}} \Phi \sqrt{\frac{\operatorname{det} \Delta^{0}}{\operatorname{det} \Delta_{\xi}^{-}}} d \mathscr{M},
$$

$$
\Delta_{\xi}^{-}=\frac{1}{2 \pi \xi^{2}} \Delta_{A}^{-}
$$

if $k \geqq 0$. (Note that $d \mathscr{M}$ is the measure of the $L^{2}$ Riemannian metric on the moduli space.) For $k<0$ we have analogous expressions, but with $\Delta^{-}$replaced by the operator $\Delta^{+}$obtained by replacing $d_{A}^{-}$with $d_{A}^{+}$in (1.3). For notational simplicity, we will henceforth write only $\Delta^{-}$when we wish to deal with all $k$ simultaneously, it being understood that when $k<0$ the minus should be replaced by a plus.

In general, the spaces $\mathscr{B}$ and $\mathscr{M}$ have singularities, and the above approach must consequently be modified. We will do this by reversing the order of the two steps above. This part of the argument assumes that $M$ is $S^{4}$ with its standard metric; we can therefore make use of the results stated in Sect. 1. 
Starting with the original integral (2.1), we first apply (2.3) on $\mathscr{A}$. By Lemma 1.2 and Eq. (2.4)

$$
\int_{\mathscr{A}} \Phi e^{-S_{1} / \xi^{2}} d \mathscr{A} \sim \int_{\mathscr{S} \mathscr{D}} \frac{\Phi}{\sqrt{\operatorname{det} \Delta_{\xi}^{-}}} e^{-S_{1} / \xi^{2}} d \mathscr{S} \mathscr{D} .
$$

Passing to the quotient by the based gauge group $\mathscr{G}^{0}$,

$$
Z(\Phi)=\int_{\tilde{M}} \frac{\Phi}{\sqrt{\operatorname{det} \Delta_{\xi}^{-}}} e^{-S_{1} / \xi^{2}} \operatorname{Vol}\left(\tilde{\mathcal{O}}_{A}\right) d \tilde{\mathscr{M}},
$$

where $\widetilde{\mathcal{O}}_{A}$ is the orbit of $\mathscr{G}^{0}$ through $A$ and $d \tilde{\mathscr{M}}$ is the volume element of the Riemannian metric $\tilde{\mathbf{g}}$ of Theorem 1.1. As before we can write $\operatorname{Vol}\left(\widetilde{\mathscr{O}}_{A}\right)$ $=\operatorname{Vol}\left(\mathscr{G}^{0}\right) \sqrt{\operatorname{det} \widetilde{\triangle}_{A}^{0}}$, where $\tilde{U}_{A}^{0}$ is an appropriate Laplacian. Thus $\operatorname{Vol}\left(\widetilde{\mathcal{O}}_{A}\right)$ is a continuous function on $\tilde{\mathscr{M}}$.

There are now two cases. First, when $k \neq 0$ Theorem 1.1 and Lemma 1.4 imply that the set of reducible connections has measure zero in $\tilde{\mathscr{M}}$, so we can replace the above integral by an integral over $\tilde{\mathscr{M}}^{*}$. This is the same as having started with an integral over $\mathscr{A}^{*}$ instead of over $\mathscr{A}$; this eliminates the singularities and we can then apply Steps 1 and 2 above to obtain, for $k \neq 0$,

$$
Z_{k}(\Phi)=\operatorname{Vol}(\mathscr{G}) e^{-8 \pi^{2}|k| / \xi^{2}} \int_{\mathscr{M}^{*}} \Phi \sqrt{\frac{\operatorname{det} \Delta^{0}}{\operatorname{det} \Delta_{\xi}^{-}}} d \mathscr{M} .
$$

The case $k=0$ is different. As $\xi \rightarrow 0$ the integrand in (2.10) concentrates at the single point $\mathscr{M}_{0}\left(S^{4}, g_{0}\right)=\theta$, which is a singular point of $\mathscr{B}$ and $\mathscr{M}$. However, $\mathscr{\mathscr { B }}$ and $\tilde{\mathscr{M}}$ are smooth at $\theta$, and formula (2.11) gives

$$
Z_{0}(\Phi)=\operatorname{Vol}\left(\widetilde{\mathcal{O}}_{\theta}\right) \frac{\Phi(\theta)}{\sqrt{\operatorname{det} \Delta_{\xi}^{-}}}
$$

where the Laplacian is that of the trivial connection $\theta$. (We can also replace $\widetilde{\mathcal{O}}_{\theta}$ here by just $\mathcal{O}_{\theta}$ since the two orbits are the same.) Understanding the volume term in this expression requires a more detailed examination of the $L^{2}$ geometry of the gauge group.

Let $\mathscr{G}=\mathscr{G}_{s+1}, s>1$, be the Sobolev gauge group on the $k=0$ principal $G$-bundle over $(M, g)$. A trivialization of this bundle defines a notion of constant gauge transformation. These transformations are included in the full gauge group as a subgroup $G_{c}$ isomorphic to $G / Z$. Letting $\bar{G}$ be the homogeneous space $\mathscr{G} / G_{c}$, we then have a fibration

$$
G_{c} \rightarrow \mathscr{G} \rightarrow \overline{\mathscr{G}} .
$$

The group $\mathscr{G}_{s+1}$ has a bi-invariant $L^{2}$ Riemannian metric obtained by identifying the left-invariant vector fields on $\mathscr{G}_{s+1}$ with its Lie algebra $L_{s+1}^{2}(\operatorname{Ad} P)$ and using the $L^{2}$ metric. There is then an induced homogeneous Riemannian metric on $\overline{\mathscr{G}}_{s+1}$ which makes the projection $\pi: \mathscr{G} \rightarrow \overline{\mathscr{G}}$ a Riemannian submersion. To define it, note that the vertical subspace of $\pi$ at $\gamma \in \mathscr{G}$ is

$$
V_{\gamma}=\left\{\gamma X \gamma^{-1} \mid X \text { is a constant section in } L_{s+1}^{2}(\operatorname{Ad} P)\right\} .
$$

Inside $L_{s+1}^{2}(\operatorname{Ad} P)$, the $L^{2}$-orthogonal complement of $V_{\gamma}$ coincides with the $L_{s+1}^{2}$-orthogonal complement; both can then be identified with $T_{\pi(\gamma)} \overline{\mathscr{G}}$, and the $L^{2}$ metric on this subspace gives the required homogeneous metric on $\overline{\mathscr{G}}$. 
Proposition 2.1. (a) The action of $\mathscr{G}_{s+1}$ on the trivial connection $\theta$ defines an inclusion $\overline{\mathscr{G}}_{s+1} \rightarrow \mathscr{A}_{s}$ which is a diffeomorphism onto the $\mathscr{G}_{s+1}$-orbit $\mathcal{O}_{\theta}$.

(b) $\operatorname{Vol}\left(G_{c}\right)=\left[\xi^{-2} \operatorname{Vol}(M, g)\right]^{d / 2}(\operatorname{Vol} G) /|Z|$, where $d$ is the dimension of $G$. In particular, for $G=S U(2)$ we have $\operatorname{Vol}\left(G_{c}\right)=16 \sqrt{2} \pi^{2} \xi^{-3}(\operatorname{Vol}(M, g))^{3 / 2}$.

(c) $\operatorname{Vol}\left(\mathcal{O}_{\theta}\right)=\left(\operatorname{Vol} \mathscr{G} / \operatorname{Vol} G_{c}\right) \sqrt{\operatorname{det}\left(\bar{\Delta}^{0}\right)}=\operatorname{Vol} \mathscr{G} \cdot 2^{-9} \cdot 3^{3 / 2} \cdot \pi^{-5} \xi^{3} \sqrt{\operatorname{det}\left(\bar{\Delta}^{0}\right)}$,

where $\bar{\Delta}^{0}$ is the restriction of $\Delta^{0}$ to the $L^{2}$-orthogonal complement of the constants in $L_{s+1}^{2}(\operatorname{Ad} P)$.

Proof. (a) For each $\gamma \in \mathscr{G}$ there is a unique constant gauge transformation $\gamma_{c} \in G_{c}$ which agrees with $\gamma$ at the point $x_{0}$. Define a map $F: \mathscr{G} \rightarrow \mathscr{G}^{0}$ by $F(\gamma)=\gamma \gamma_{c}^{-1}$; this descends to a map $F: \bar{G} \rightarrow \mathscr{G}^{0}$. The action of $\gamma$ on $\theta$, given by $i(\gamma)=\gamma \cdot \theta=\theta+\gamma d \gamma^{-1}$, also descends to give an injective map $\bar{i}: \overline{\mathscr{G}} \rightarrow \mathscr{A}$ onto the gauge orbit $\widetilde{\mathcal{O}}_{\theta}=\mathcal{O}_{\theta}$. Its differential at $\gamma$ is

$$
\gamma \circ d \circ \gamma^{-1}: V_{\gamma}^{\perp} \subset L_{s+1}^{2}(\operatorname{Ad} P) \rightarrow T_{\gamma \cdot \theta} \mathcal{O}_{\theta}
$$

this is smooth and invertible, so by the inverse function theorem $\bar{i}$ is a diffeomorphism.

(b) The $L^{2}$ metric on $\mathscr{G}$ is bi-invariant, so its restriction to $G_{c}$ is a multiple of the Killing form $K$. It is easy to check that this restricted metric is $\frac{-V}{\xi^{2}} K$, where
$V=\operatorname{Vol}(M, g)$, and hence

$$
\operatorname{Vol}\left(G_{c}\right)=\left(\frac{V}{\xi^{2}}\right)^{d / 2} \operatorname{Vol}(G / Z),
$$

where $\operatorname{Vol}(G / Z)=(\operatorname{Vol} G) /|Z|$ is measured with respect to the Killing form. For $G=S U(2)$ we have $|Z|=2$, and under the usual identification of $S U(2)$ with the unit quaternions the Killing form is -8 times the standard metric on $S^{3}$. Hence $\operatorname{Vol}(G / Z)=\frac{1}{2} \cdot 8^{3 / 2} \cdot 2 \pi^{2}$, and $\operatorname{Vol}\left(G_{c}\right)$ is as stated.

(c) From part (a) we have $\operatorname{Vol}\left(\widetilde{\mathcal{O}}_{\theta}\right)=\sqrt{\operatorname{det}\left(\bar{\Delta}^{0}\right)} \operatorname{Vol}(\overline{\mathscr{G}})$, and since (2.14) is a Riemannian submersion $\operatorname{Vol} \overline{\mathscr{G}}=\operatorname{Vol} \mathscr{G} / \operatorname{Vol} G_{c}$. The final formula then follows from part (b) since $\operatorname{Vol}\left(S^{4}\right)=8 \pi^{2} / 3$.

From Proposition 2.1 we obtain, at last, the semiclassical term at the trivial connection:

$$
Z_{0}(\Phi)=\operatorname{Vol} \mathscr{G} \frac{3^{3 / 2} \xi^{3}}{512 \pi^{5}} \Phi(\theta) \sqrt{\frac{\operatorname{det} \bar{\Delta}^{0}}{\operatorname{det} \Delta_{\xi}^{-}}}
$$

We now have expressions - Eqs. (2.12) and (2.15) - for the semiclassical partition functions which include the effects of the singularities in the moduli spaces of $S U(2)$ connections on the standard 4-sphere. These give a revised expression for the ratio relevant to the semiclassical expectation:

$$
\begin{aligned}
\frac{Z_{k}(\Phi)}{Z_{0}(1)}= & 512 \cdot 3^{-3 / 2} \pi^{5} \xi^{-3}\left(\frac{\operatorname{Vol}\left(\mathscr{G}_{k}\right)}{\operatorname{Vol}\left(\mathscr{G}_{0}\right)}\right) C_{0}(\xi)^{-1} e^{-8 \pi^{2}|k| \xi^{-2}} \\
& \times \int_{\mathscr{M}_{k}^{*}} \Phi \sqrt{\frac{\operatorname{det} \Delta^{0}}{\operatorname{det} \Delta_{\xi}^{-}}} d \mathscr{M},
\end{aligned}
$$

where $\mathscr{G}_{k}$ is the gauge group of the bundle with instanton number $k$ and $C_{0}(\xi)$ is the last factor in (2.15) - the square root of the determinant ratio for the Laplacians of 
the trivial connection. This formula becomes conceptually and notationally simpler after making three remarks.

- We will assume that the $\operatorname{ratio} \operatorname{Vol}\left(\mathscr{G}_{k}\right) / \operatorname{Vol}\left(\mathscr{G}_{0}\right)$ is 1 . This is implicit in the "gauge-fixing" approach to path integrals usually taken by physicists (see [BV] and Appendix II of [Sc]).

- By (2.15) the expectation of an observable on $\mathscr{B}_{0}$ is simply $Z(\Phi) / Z(1)=\Phi(\theta)$, independent of $\xi$.

- The integral in (2.16) is best thought of as the integral of $\Phi$ with respect to the "semiclassical measure"

$$
d v_{\xi}=\sqrt{\frac{\operatorname{det} \Delta^{0}}{\operatorname{det} \Delta_{\xi}^{-}}} d \mathscr{M}
$$

on the moduli space. This measure effectively accounts for the leading-order quantum fluctuations around the classical solutions.

We thus arrive at our final expression for the semiclassical expectation: for any observable $\Phi$ that vanishes on $\mathscr{B}_{l}$ for $|l|<|k|$ we have

$$
\begin{aligned}
\langle\Phi\rangle_{\mathrm{sc}} & =\frac{Z_{k}(\Phi)+Z_{-k}(\Phi)}{Z_{0}(1)} \\
& =\left\{\begin{array}{lll}
\Phi(\theta) & \text { if } & k=0 \\
512 \cdot 3^{-3 / 2} \pi^{5} \xi^{-3} C_{0}(\xi)^{-1} e^{-8 \pi^{2}|k| / \xi^{2}} \int_{\mathscr{M}^{*}{ }_{k} \cup \mathcal{M}_{k}^{*}} \Phi d v_{\xi} & \text { if } & k \neq 0,
\end{array}\right.
\end{aligned}
$$

provided this number does not vanish. Notice that these semiclassical expectations are integrals over finite-dimensional Riemannian manifolds. Hence the problem of defining the measures and the infinite-dimensional integrals in (2.1) and (2.2) disappears after one makes the semiclassical approximation. In fact, for any observable that vanishes on $\mathscr{B}_{0}$ but not on $\mathscr{B}_{1}$, the semiclassical expectation will be completely calculable once we have an explicit formula for the semiclassical measure (2.17).

\section{Zeta Functions}

The goal of the next several sections is to determine the semiclassical measure $d v$ explicitly on the moduli space $\mathscr{M}_{1}\left(S^{4}\right)$. We will do this using $\zeta$-function methods. We begin below by recalling the definition of determinants and giving two basic lemmas. We then describe our approach to computing determinants as functions on the moduli space. In the rest of this section we concentrate on the determinant factor

$$
\sqrt{\operatorname{det}\left(\Delta_{A}^{0}\right) / \operatorname{det}\left(\Delta_{A}^{-}\right)},
$$

showing how the computation reduces to finding certain heat kernel coefficients. (These coefficients will be identified in Sect. 4.) The key observation (Lemma 3.4) is that the families of operators $\left\{\Delta_{A}^{p}\right\}$ can be replaced by families $\left\{\widetilde{U}_{v}^{p}\right\}$ in which the connection is fixed but the metric varies.

Let $D: \Gamma(E) \rightarrow \Gamma(E)$ be a self-adjoint, non-negative elliptic operator on a vector bundle $E$ over a compact Riemannian manifold $M^{n}, n$ even. (Later in this section we will specialize to $n=4$.) By standard elliptic theory $L^{2}(E)$ has a complete orthonormal basis of eigensections $\left\{\phi_{i} \mid D \phi_{i}=\lambda_{i} \phi_{i}\right\}$; the spectrum $\left\{\lambda_{i}\right\}$ is discrete and non-negative and each eigenspace is finite-dimensional. We will make frequent use of two objects associated with $D$ : its heat family and its zeta function. 
The heat family associated with $D$ is the unique family of operators $H_{t}$ on $L^{2}(E)$ satisfying the heat equation

$$
\frac{\partial}{\partial t}\left(H_{t}\right)=-D H_{t}
$$

with $H_{0}=$ identity, and hence is often written $H_{t}=e^{-t D}$. Some basic properties of the heat kernel are listed below; all are consequences of the standard construction described in [P] and [RaSi], and the parabolic PDE techniques found in Chap. 9 of $[\mathrm{F}]$.

(a) For every $t>0$ and every integer $k \geqq 0, H_{t}$ is a bounded operator from $L^{2}(E)$ to the Sobolev space $L_{k}^{2}(E)$ of sections whose first $k$ derivatives are in $L^{2}$. Viewed as an operator from $L_{k}^{2}(E)$ to itself, $H_{t}$ is trace-class.

(b) $H_{t}$ is given by convolution with the heat kernel

$$
K_{D}(t, x, y)=\sum_{i} e^{-t \lambda_{i}} \phi_{i}(x) \phi_{i}^{*}(y) d y \in E_{x} \otimes E_{y}^{*} \otimes \Lambda_{y}^{n} T^{*} M,
$$

where $\phi_{i}^{*}(y)=\left(\phi_{i}(y), \cdot\right)$ and $d y$ is the Riemannian volume form in the last variable. The sum converges uniformly on $\{\varepsilon \leqq t<\infty\}$ for any $\varepsilon>0$. The (smooth) density $K_{D}$ is the unique section satisfying

$$
\begin{cases}\left(\partial_{t}+D_{x}\right) K_{D}(t, x, y)=0, & t>0 \\ \lim _{t \rightarrow 0} \int_{M} K_{D}(t, x, y) \psi(y)=\psi(x), & \forall \psi \in \Gamma(E) .\end{cases}
$$

(c) Let $\varrho>0$ be a smooth function on $M \times M$ that agrees with the distance function $d$ in neighborhood of the diagonal. Then for each $l>0$ we can write

$$
K_{D}(t, x, y)=P_{l}(t, x, y)+R_{l}(t, x, y) .
$$

Here the parametrix $P_{l}$ is a smooth double-form on $\mathbf{R}^{+} \times M \times M$ given by

$$
P_{l}(t, x, y)=(4 \pi t)^{-\frac{n}{2}}\left(1+\sum_{k=1}^{l} t^{k} a_{k}(x, y)\right) e^{-\frac{Q^{2}(x, y)}{4 t}}
$$

for certain forms $a_{k}(x, y)$. The remainder $R_{l}$ and its derivatives satisfy the following bounds: for each multi-index $\alpha$ of order $m \geqq 0$ on $M \times M$ there are positive constants $c$ and $C$ such that

$$
\left|\nabla^{\alpha} R_{l}(t, x, y)\right| \leqq C t^{l+1-\frac{m+n}{2}} e^{-c \frac{Q^{2}(x, y)}{t}}
$$

for $0 \leqq t \leqq 1$.

(d) $K_{D}, R_{l}$, and each of the coefficients $a_{k}(x, y)$ depend smoothly on the coefficients of $D$.

In addition, there are formulae for the coefficients $a_{k}(x, y)$. Some of these will be computed in Sect. 4.

Below, it will often be convenient to separate the action of $H_{t}$ on $\operatorname{ker}(D)$ (where it acts as the identity) from its action on the orthogonal complement $\operatorname{ker}(D)^{\perp}$. If $\left\{\phi_{i}^{0}\right\}$ is an $L^{2}$-orthonormal basis of $\operatorname{ker}(D)$, then $H_{t}$ acts on $\operatorname{ker}(D)^{\perp}$ by convolution with the kernel

$$
K_{D}^{+}(t, x, y)=K_{D}(t, x, y)-\left(\sum \phi_{i}^{0}(x)\left(\phi_{i}^{0}(y)\right)^{*}\right) d y .
$$


Since the last term in (3.7) is independent of $t, K_{D}^{+}$has a parametrix $P_{l}^{+}$given by (3.5) with coefficients $a_{k}^{+}(x, y)=a_{k}(x, y)$ for $k \neq n / 2$, and for $k=n / 2$ we have

$$
a_{\frac{n}{2}}^{+}(x, y)=a_{\frac{n}{2}}(x, y)-\sum \phi_{i}^{0}(x)\left(\phi_{i}^{0}(y)\right)^{*} d y .
$$

Let $D^{+}$denote the restriction of $D$ to $\operatorname{ker}(D)^{\perp}$. For $s \in \mathbf{C}$ with $\operatorname{Re}(s)$ sufficiently large, $\left(D^{+}\right)^{-s}$ is trace-class. For such $s$, we define the $\zeta$-function of $D$ by

$$
\zeta_{D}(s)=\sum_{\lambda_{i}>0} \lambda_{i}^{-s}=\operatorname{Tr}\left(\left(D^{+}\right)^{-s}\right) ;
$$

by the results of Seeley [S], the sum converges for $\operatorname{Re}(s) \gg 0$ and has a meromorphic continuation to the entire s-plane with only simple poles. Applying the Mellin transform, we have

$$
\begin{aligned}
\zeta_{D}(s) & =\frac{1}{\Gamma(s)} \int_{0}^{\infty} t^{s-1} \operatorname{Tr}\left(e^{-t D^{+}}\right) d t \\
& =\frac{1}{\Gamma(s)} \int_{0}^{\infty} t^{s-1}\left[\int_{M} \operatorname{tr}\left(\imath^{*} K_{D}^{+}(t)\right] d t\right.
\end{aligned}
$$

for $\operatorname{Re}(s) \gg 0$. [Here $\left(i^{*} K_{D}(t)\right)(x)=K_{D}(t, x, x) d x$, the restriction of $K_{D}$ to the diagonal.] Motivated by the observation that $-\zeta^{\prime}(0)$ is formally $\sum \log \lambda_{i}$, one defines the determinant of $D$ by

$$
\operatorname{det}(D)=\left(\prod_{\lambda_{i} \leqq 0} \lambda_{i}\right) e^{-\zeta^{\prime}(0)} .
$$

Note in particular that if $D$ has a zero eigenvalue then $\operatorname{det}(D)=0$. (In our case we are assuming that $D$ has no strictly negative eigenvalues, but the formula above works equally well in the more general case of operators with a finite negative spectrum, provided the definition of $D^{+}$is suitably modified.)

The next lemma gives some simple but important properties of the heat kernel and the $\zeta$-function determinant.

Lemma 3.1. (a) For any $t>0$, and any zeroth-order invertible differential operator $L: \Gamma(E) \rightarrow \Gamma(E)$, we have $\operatorname{Tr}\left(L^{-1} e^{-t D} L\right)=\operatorname{Tr}\left(e^{-t D}\right)$. Moreover, $\zeta_{L^{-1} D L}(s)=\zeta_{D}(s)$ for all $s$, and $\operatorname{det}\left(L^{-1} D L\right)=\operatorname{det}(D)$.

(b) For any $c>0, \operatorname{det}(c D)=c^{\zeta(0)} \operatorname{det}(D)$.

Proof. (a) It follows immediately from the existence and uniqueness theorem for the defining Eq. (3.2) that the heat operator of $L^{-1} D L$ is $L^{-1} e^{-t D} L$; thus by (3.9),

$$
\zeta_{L^{-1} D L}(s)=\frac{1}{\Gamma(s)} \int_{0}^{\infty} t^{s-1}\left[\operatorname{Tr}\left(L^{-1} e^{-t D} L\right)-\operatorname{dim} \operatorname{ker}\left(L^{-1} D L\right)\right] d t .
$$

Commuting inside the trace (see Lemma A.2), we have

$$
\operatorname{Tr}\left(L^{-1} e^{-t D} L\right)=\operatorname{Tr}\left(L L^{-1} e^{-t D}\right)=\operatorname{Tr}\left(e^{-t D}\right) ;
$$

moreover, $\operatorname{dim} \operatorname{ker}\left(L^{-1} D L\right)=\operatorname{dim} \operatorname{ker} D$. Thus $\zeta_{L^{-1} D L}(s)=\zeta_{D}(s)$ for $\operatorname{Re}(s) \gg 0$, and hence for all $s$ by analytic continuation. Therefore $\operatorname{det}\left(L^{-1} D L\right)=\operatorname{det}(D)$.

(b) Substituting into (3.9) and changing variables by $\tau=c t$ shows that $\zeta_{c D}(s)$ $=c^{-s} \zeta_{D}(s)$ for $\operatorname{Re}(s) \gg 0$, and hence for all $s$. But then $\zeta_{c D}^{\prime}(0)=-(\log c) \zeta(0)+\zeta^{\prime}(0)$, and the result follows from the definition (3.9).

Note that because of (b), the number $\zeta(0)$ should be interpreted as the formal dimension of the Hilbert space on which $D$ acts. The next lemma shows how $\zeta(0)$ can be calculated from the heat kernel coefficients. 
Lemma 3.2. Let $D$ be as above, and let $a_{k}$ be the coefficients of the parametrix (3.5). Then

$$
\zeta_{D}(0)=\left[(4 \pi)^{-\frac{n}{2}} \int_{M} \operatorname{tr}\left(\imath^{*} a_{n / 2}\right)\right]-\operatorname{dim} \operatorname{ker} D .
$$
Proof. Let $B_{D}(s)=\Gamma(s) \zeta_{D}(s)$. Since $\zeta_{D}$ is analytic at $s=0$, we have $\zeta_{D}(0)=\underset{s=0}{\operatorname{Res}}\left(B_{D}(s)\right)$.
By (3.9), $B_{D}(s)$ is the analytic continuation of

$$
\int_{0}^{1} t^{s-1}\left[\int_{M} \operatorname{tr}\left(\imath^{*} K_{D}^{+}(t)\right)\right] d t+\int_{1}^{\infty}\left[\int_{M} \operatorname{tr}\left(\imath^{*} K_{D}^{+}(t)\right)\right] d t .
$$

Lemma A.1 in the appendix shows that the second of these integrals is an entire function of $s$, so does not contribute to the residue. Substituting the parametrix $P_{l}^{+}$ of $K_{D}^{+}$with $l=n / 2$ into the first integral and explicitly integrating the terms in the summation gives

$$
\zeta_{D}(0)=\operatorname{Res}_{s=0}\left\{\left[\sum_{k=0}^{n / 2} \frac{(4 \pi)^{-n / 2}}{k+s-n / 2} \int_{M} t^{*} a_{k}^{+}\right]+\int_{0}^{1} t^{s-1} \int_{M} \phi(\cdot, t) d t\right\}
$$

with $\phi(x, t)=O(t)$ uniformly in $x$ as $t \rightarrow 0$. Again, Lemma A.1 shows that the integral involving $\phi$ is holomorphic for $\operatorname{Re}(s)>-1$, so the only contribution to the residue comes from the $k=n / 2$ term. Finally, Eq. (3.8) implies

$$
\operatorname{tr}\left(a_{n / 2}^{+}(x, x)\right)=\operatorname{tr}\left(a_{n / 2}(x, x)\right)-\sum\left|\phi_{i}^{0}(x)\right|^{2} d x .
$$

We thus obtain (3.12).

Remark. Since the coefficients $a_{k}(D)$ are continuous functions of the coefficients of $D$, it is apparent from (3.12) that if we vary $D$, the number $\zeta_{D}(0)$ jumps by 1 if we cross a zero eigenvalue. This also follows quickly just from the definition of $\zeta(s)$. But despite the discontinuity in $\zeta_{D}(0)$ at such $D$, the determinant $\operatorname{det}(D)$ is still continuous. For if $\varepsilon(D)$ is the smallest positive eigenvalue of $D$ and $\operatorname{det}^{\prime}(D)$ denotes the determinant obtained by excluding $\varepsilon$ from the spectrum, we have $\operatorname{det}(D)$ $=\varepsilon(D) \operatorname{det}^{\prime}(D)$, and thus as $\varepsilon(D) \rightarrow 0$ so $\operatorname{does} \operatorname{det}(D)$.

The operators whose $\zeta$-functions we will analyze in this section are the Laplacians given in (1.3). As $A$ varies, $\Delta_{A}^{p}$ and $\Delta_{A}^{-}$are families of elliptic operators parametrized by the space of connections. Since their eigenvalues are gaugeinvariant, their zeta-functions and determinants descend to functions on $\mathscr{B}=\mathscr{A} / \mathscr{G}$. The simplest such function is $\zeta(0)$; it is given by (3.12), where the $a_{n / 2}$ are regarded as functions of $A$. For $n=4$ the value of $a_{2}(x, x)$ is calculated in Sect. 4 , giving the following corollary.

Corollary 3.3. (a) Let $D$ be one of the operators $\Delta_{A}^{i}$ or $\Delta_{A}^{-}$. Then $\zeta_{D}(0)$ is constant along the smooth part $\mathscr{M}^{*}$ of each component of the moduli space.

(b) For any self-dual connection $A$ the $\zeta$-functions $\zeta_{0}, \zeta_{1}$, and $\zeta_{-}$of $\Delta_{A}^{0}, \Delta_{A}^{1}$, and $\Delta_{A}^{-}$ are related by $\zeta_{1}=\zeta_{0}+\zeta_{-}$.

Proof. (a) On the smooth part of $\mathscr{M}$ we have $\operatorname{dim} \operatorname{ker} \Delta_{A}^{0} \equiv \operatorname{dim} \operatorname{ker} \Delta_{A}^{-} \equiv 0$ and $\operatorname{dim} \operatorname{ker} \Delta_{A}^{1} \equiv \operatorname{dim} \mathscr{M}$. We can then apply Lemmas 3.2 and 4.1 (see Sect. 4 below), taking $A$ to be either self-dual or anti-self-dual. In all cases $\zeta_{D}(0)$ is a constant (whose value depends on the Riemannian geometry of $M$ ) plus a multiple of the Yang-Mills action (1.6). Since the action is itself constant along $\mathscr{M}$, we conclude that so is $\zeta_{D}(0)$. 
(b) In the elliptic complex (1.2) the operators $d_{A}^{*}$ and $d_{A}^{-}$intertwine the eigenspaces of $\Delta_{A}^{1}$ corresponding to positive eigenvalues with the positive eigenspaces of $\Delta_{A}^{0}$ and $\Delta_{A}^{-}$, so that

$$
\operatorname{Spec}^{+}\left(\Delta_{A}^{1}\right)=\operatorname{Spec}^{+}\left(\Delta_{A}^{0}\right) \cup \operatorname{Spec}^{+}\left(\Delta_{A}^{-}\right) .
$$

(By Spec we mean the set of eigenvalues together with their multiplicities.) Now for a general nonnegative self-adjoint elliptic operator $D$ we have $\operatorname{Tr}\left(e^{-t D^{+}}\right)=\sum e^{-t \lambda_{n}}$ for $t>0$, where $\left\{\lambda_{n}\right\}$ are the positive eigenvalues of $D$. Hence

$$
\operatorname{Tr}\left(e^{-t\left(\Delta_{A}^{1}\right)^{+}}\right)=\operatorname{Tr}\left(e^{-t\left(\Delta_{A}^{0}\right)^{+}}\right)+\operatorname{Tr}\left(e^{-t\left(\Delta_{\bar{A}}\right)^{+}}\right) .
$$

The Mellin transform (3.9) then gives the corresponding addition formula for the $\zeta$-functions.

We are interested in computing the determinant ratio (3.1) on the moduli space $\mathscr{M} \subset \mathscr{B}$, but for a general moduli space this seems to be a difficult problem. Therefore we now specialize to the moduli space $\mathscr{M}_{1}\left(S^{4}\right)$, the space of 1-instantons over the four-sphere. In this case we can explicitly compute the function $\operatorname{det} \Delta_{A}^{0} / \operatorname{det} \Delta_{A}^{-}$on $\mathscr{M}$ and examine its asymptotics near the boundary of $\mathscr{M}$. Our approach is as follows. The explicit description of $\mathscr{M}$ given below shows that there is a "center point" $\left[A_{0}\right] \in \mathscr{M}$ and a diffeomorphism $\mathscr{M} \cong \mathbf{R}^{5}$ which carries $\left[A_{0}\right]$ to 0 and intertwines an isometric action of $S O(5)$ with the usual action on $\mathbf{R}^{5} ; \operatorname{det} \Delta_{A}^{i}$ is invariant under this $S O(5)$-action. Hence it suffices to determine the function $\operatorname{det} \Delta_{A}^{0} / \operatorname{det} \Delta_{A}^{-}$along the radial line from $\left[A_{0}\right]$. We will do this by calculating the radial derivative $\delta \log \operatorname{det}\left(\Delta_{A}^{0} / \operatorname{det} \Delta_{A}^{-}\right)$and integrating.

To carry out this program, we first review the parametrization of $\mathscr{M}_{1}\left(S^{4}\right)$ given in [GP 1]. In this case $P$ is the bundle of orthonormal frames of $\Lambda_{+}^{2}\left(T S^{4}\right)$, and we assume $S^{4}$ has its standard metric $g$. The Levi-Civita connection on $S^{4}$ induces a self-dual connection $A^{0}$ on $P$, whose gauge-orbit we review as the center point of $\mathscr{M}$. We let $d_{0}$ denote the corresponding covariant derivative on $\Lambda_{+}^{2}\left(T S^{4}\right)$. The group $\operatorname{Conf}\left(S^{4}\right) \cong S O(5,1)$ of conformal diffeomorphisms of $S^{4}$ acts on $P$, hence on $\mathscr{A}$, carrying one self-dual connection into another. This action commutes with that of the gauge group, so there is an induced action on $\mathscr{M}$, which is shown in [AHS, Theorem 9.1] to be transitive. Hence $\mathscr{M} \cong S O(5,1) / \operatorname{Stab}\left(\left[A^{0}\right]\right)$. Now, if $\Phi \in \operatorname{Conf}\left(S^{4}\right)$ and $\Phi^{*} g=\gamma^{2} g$, the covariant derivative on $\Lambda_{+}^{2}\left(T S^{4}\right)$ corresponding to the image of $A^{0}$ under $\Phi$ is $\gamma^{2}\left(\Phi^{*} d_{0}\right) \gamma^{-2}$. (Up to conjugation, this is the Levi-Civita connection of the metric $\Phi^{*} g$.) It follows that $\operatorname{Stab}\left(A^{0}\right)$ is precisely the isometry group $S O(5)$ of $S^{4}$. Therefore $\mathscr{M} \cong S O(5,1) / S O(5) \cong \mathbf{R}^{5}$.

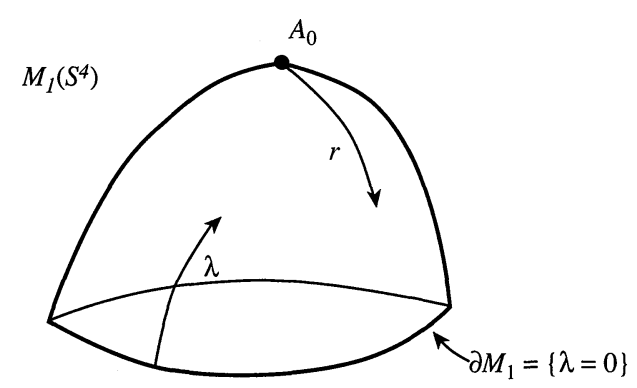


To coordinatize $\mathscr{M}$, we use a distinguished 5-parameter family of conformal transformations of $S^{4}$, obtained as follows. Each $v \in \mathbf{R}^{5}$ determines a linear function on $\mathbf{R}^{5}$ (inner product with $v$ ), which restricts to a function $f_{v}$ on $S^{4} \subset \mathbf{R}^{5}$. The vector field $X_{v}=-\operatorname{grad}\left(f_{v}\right)$ generates a 1-parameter subgroup of $\operatorname{Conf}\left(S^{4}\right)$. Let $\Phi_{v}$ denote the unit-time flow of $X_{v}$. Then $\left\{\Phi_{v} \mid v \in \mathbf{R}^{5}\right\}$ is a submanifold of $S O(5,1)$ which, one can show, projects diffeomorphically to the quotient $S O(5,1) / S O(5)$. The obvious coordinate system on this submanifold therefore pulls back to a coordinate system on $\mathscr{M}$. (This is the coordinate map $\bar{Q}$ introduced in Sect. 4 of [GP 1].)

Let $A_{v} \in \mathscr{A}$ be the connection corresponding to $v \in \mathbf{R}^{5}$ as above and let $d_{v}$ be the associated covariant derivative on $\operatorname{Ad} P$. We will abbreviate $\Delta_{A_{v}}^{p}$ as $\Delta_{v}^{p}$, etc. We will also consider the Laplacians $\widetilde{\Delta}_{v}^{p}$ and $\tilde{\Delta}_{v}^{-}$formed from $d_{0}$, but with adjoints computed with respect to the metric $\Phi_{v}^{*} g$ instead of $g$.

Lemma 3.4. For $p=0,1$, or - ,

$$
\operatorname{det}\left(\Delta_{v}^{p}\right)=\operatorname{det}\left(\tilde{\Delta}_{-v}^{p}\right) \text {. }
$$

Proof. For $p=0,1,2$ let $\square_{v}^{p}=d_{v} d_{v}^{*}+d_{v}^{*} d_{v}$ be the usual covariant Laplacian on $p$-forms. By definition we have $d_{v}=\gamma_{v}^{2}\left(\Phi_{v}^{*} d_{0}\right) \gamma_{v}^{-2}$. Now $d_{v}^{*}= \pm * d_{v} *$, where $*$ is the Hodge star-operator associated to the metric $g$. Noting that $*=\Phi_{v}^{*}\left(*_{\left(\Phi_{v}^{-1}\right)^{*} g}\right)$ $=\Phi_{v}^{*}\left(*_{-v}\right)$ by the naturality of $*$, we have $d_{v}^{*}=\gamma_{v}^{2}\left(\Phi_{v}^{*}\left(d_{0}^{*-v}\right)\right) \gamma_{v}^{-2}$. Hence

$$
\square_{v}^{p}=\gamma_{v}^{2}\left(\Phi_{v}^{*} \square_{-v}^{p}\right) \gamma_{v}^{-2} \text {, }
$$

where $\Phi^{*} \Delta=\Phi_{*}^{-1} \circ \Delta \circ \Phi_{*}$. Lemma 3.1 a now immediately implies the $p=0$ case of (3.13). We have already seen in Eqs. (1.3-4) that $\Delta_{v}^{-}$is the restriction to $\Omega_{-}^{2}$ of $\square_{v}^{2}$; the same is true with tildes inserted since $*$ acting on 2 -forms is conformally invariant. Hence the $p=-$ case of (3.13) follows. The case $p=1$ is similar and we omit the details.

Lemma 3.4 shows that we can replace the Laplacians $\Delta$ by the Laplacians $\tilde{\Delta}$ without changing the determinants or zeta functions. The value of this is that the variations of the latter are mostly easily calculated. [Here we mean variation in the weakest sense: $(\delta \Delta)(f) \stackrel{\text { def }}{=} \delta(\Delta f)$. In the next several lemmas we will compute these variations $\delta \tilde{\Delta}$ and relate them to the variation of the determinant ratio (3.1). The more technical points in these arguments are given as lemmas in the appendix.

Lemma 3.5. Fix $v \in \mathbf{R}^{5}$ and consider the 1-parameter families $D_{\beta}^{0^{\text {def }}=} \tilde{\Delta}_{-\beta v}^{0}$ and $D_{\beta}^{-}=\tilde{\Delta}_{-\beta v}^{-}$. Then the variations $\delta=\left.\frac{d}{d \beta}\right|_{\beta=1}$ of these operators can be expressed in terms of the function $\psi_{-v}=\delta \log \gamma_{-\beta v} b y$

$$
\delta D^{0}=-4 \psi_{-v} D_{1}^{0}+2 d_{0}^{* \prime} \psi_{-v} d_{0}
$$

and

$$
\delta D^{-}=-4 d_{0}^{-} \psi_{-v}\left(d_{0}^{-}\right)^{* \prime},
$$

where ${ }^{* \prime}$ denotes adjoint with respect to the metric $g^{\prime}=\Phi_{-v}^{*} g=\gamma_{-v}^{2} g$.

Proof. On $p$-forms we have $*^{\prime}=\gamma_{-v}^{4-2 p} *$, implying $d_{0}^{*^{\prime}}=\gamma_{-v}^{2 p-6} \circ d_{0}^{*} \circ \gamma_{-v}^{4-2 p}$. By definition we then have

$$
D_{\beta}^{0}=\gamma_{-\beta v}^{-4} \circ d_{0}^{*} \circ \gamma_{-\beta v}^{2} \circ d_{0} \text { and } D_{\beta}^{-}=2 d_{0}^{-} \circ \gamma_{-\beta v}^{-2} \circ\left(d_{0}^{-}\right)^{*}
$$


Differentiating now yields the results.

By definition the variation in the determinant ratio (3.1) is

$$
\delta \log \sqrt{\frac{\operatorname{det} \Delta_{A}^{0}}{\operatorname{det} \Delta_{A}^{-}}}=\frac{1}{2}\left[\delta \zeta_{\Delta^{\prime}}^{\prime}(0)-\delta \zeta_{\Delta^{-}}^{\prime}(0)\right] .
$$

For any of our families of second-order linear elliptic operators $D_{\beta}\left(\right.$ e.g. $\left.\Delta_{\beta v}^{0}\right)$ set

$$
B_{\beta}(s)=\Gamma(s) \zeta_{D_{\beta}}(s) \text {. }
$$

Lemma A.6 proves that the variation $\delta=\left.\frac{\delta}{\delta \beta}\right|_{\beta=1}$ of this is given by the formula one would expect from (3.16), namely

$$
(\delta B)(s)=\delta\left(\zeta_{D}(0)\right)\left(\frac{1}{s}-\gamma\right)+\delta\left(\zeta_{D}^{\prime}(0)\right)+O(s),
$$

where $\gamma$ is Euler's constant. On the moduli space the first term vanishes by Corollary 3.3a, so

$$
(\delta B)(0)=\delta\left(\zeta_{D}^{\prime}(0)\right)
$$

and in particular

$$
\delta \log \sqrt{\frac{\operatorname{det} \Delta_{A}^{0}}{\operatorname{det} \Delta_{A}^{-}}}=\frac{1}{2}\left[\delta B_{\Delta^{0}}(0)-\delta B_{\Delta^{-}}(0)\right] .
$$

Henceforth we will abbreviate $B_{\Delta^{0}}$ and $B_{\Delta^{-}}$as $B^{0}$ and $B^{-}$, respectively.

Lemma 3.6. For $\operatorname{Re}(s)$ sufficiently large,

$$
\frac{1}{2}\left(\delta B^{-}(s)-\delta B^{0}(s)\right)=-\int_{0}^{\infty} t^{s}\left[\frac{d}{d t} \operatorname{Tr}\left(\psi_{-v}\left[e^{-t \tilde{\Delta}^{1} v}-2 e^{-t \tilde{\Delta}^{0} v}\right]\right)\right] d t .
$$

Proof. Lemmas 3.1a and 3.4 imply that the functions $B^{i}$ do not change if we replace $\Delta_{\beta v}^{i}$ by $D_{\beta}^{i}=\widetilde{L}_{-\beta v}^{i}$. We can then use (3.9) to express $B_{\beta}(s)$ as an integral and take the variation. Lemma A.5 shows that the variation can be moved inside the integral and the trace, yielding

$$
\delta B^{i}(s)=-\int_{0}^{\infty} t^{s} \operatorname{Tr}\left(\left(\delta D^{i}\right) e^{\left.-t D_{\beta}^{i}\right)}\right) d t .
$$

Writing $D^{i}$ for $D_{1}^{i}$ and applying Lemma 3.5, we then have

$$
\begin{aligned}
\frac{1}{2}\left(\delta B^{-}(s)-\delta B^{0}(s)\right)= & -\int_{0}^{\infty} t^{s} \operatorname{Tr}\left[-4 d_{0}^{-} \psi_{-v}\left(d_{0}^{-}\right)^{*^{\prime}} e^{-t D^{-}}\right. \\
& \left.+4 \psi_{-v} D^{0} e^{-t D^{0}}-2 d_{0}^{*^{\prime}} \psi_{-v} d_{0} e^{-t D^{0}}\right] d t .
\end{aligned}
$$

We may apply Lemma A.2 to commute inside the trace, rewriting the first term as $\operatorname{Tr}\left[-4 \psi_{-v}\left(d_{0}^{-}\right) *^{*} e^{-t D^{-}} d_{0}^{-}\right]$. Now, from the definition of $D^{i}$ one has $D^{-} d_{0}^{-}=d_{0}^{-} D^{1}$, and it follows easily that $e^{-t D^{-}} d_{0}^{-}=d_{0}^{-} e^{-t D^{1}}$. Hence the factors can be reordered again to obtain $\operatorname{Tr}\left[-4 \psi_{-v}\left(d_{0}^{-}\right)^{*} d_{0}^{-} e^{-t D^{1}}\right]$. Similarly reordering the factors in the third term inside the trace and combining with the first term, we obtain

$$
\begin{aligned}
-2 \operatorname{Tr}\left[\psi_{-v}\left(2\left(d_{0}^{-}\right)^{*^{\prime}} d_{0}+d_{0}^{-}\left(d_{0}^{-}\right)^{*^{\prime}}\right) e^{-t D_{1}}\right] & =-2 \operatorname{Tr}\left(\psi_{-v} D^{1} e^{-t D^{1}}\right) \\
& =2 \frac{d}{d t} \operatorname{Tr}\left(\psi_{-v} e^{-t D^{1}}\right),
\end{aligned}
$$


where we have used Lemma A.3a in the last step. The remaining term in (3.21) is also a $t$-derivative, and the result follows.

Lemma 3.7. Let $D$ be any of the Laplacians considered in the previous lemma. Then

$$
\operatorname{Tr}\left(\psi_{-v} e^{-t \tilde{D}_{-v}}\right)=-\operatorname{Tr}\left(f_{v} e^{-t D_{v}}\right) .
$$

Proof. Lemma A.3b shows that $\operatorname{Tr}\left(\psi_{-v} e^{-t D}\right)=\int_{M} \psi_{-v} \operatorname{tr}\left(\imath^{*} K_{D}\right)$, and the uniqueness of the heat kernel can be used to show that $K_{\Phi^{*} D}=\Phi^{*} K_{D}$. Hence

$$
\operatorname{Tr}\left(\psi_{-v} e^{-t D}\right)=\int_{M} \psi_{-v} \operatorname{tr}\left(\imath^{*} K_{D}\right)=\int_{M} \Phi^{*}\left(\psi_{-v}\right) \operatorname{tr}\left(\imath^{*} K_{\Phi_{v}^{*} D}\right) .
$$

Again, uniqueness shows that $\imath^{*} K_{\gamma D_{\gamma^{-1}}}=\imath^{*} K_{D}$, so

$$
\operatorname{tr}\left(K_{\Phi_{v}^{*} D}\right)=\operatorname{tr}\left(K_{\gamma_{v}^{2}\left(\Phi_{v}^{*} D\right) \gamma \bar{v}}\right) .
$$

Applying this to $D=\widetilde{\Delta}_{-v}^{p}$, and using Lemma A.3, we have

$$
\operatorname{Tr}\left(\psi_{-v} e^{-t \tilde{\Delta}_{-v}^{p}}\right)=\int_{M}\left(\Phi^{*} \psi_{-v}\right) \operatorname{tr}\left(\imath^{*} K_{\Delta_{v}^{p}}\right)=\operatorname{Tr}\left(\left(\Phi^{*} \psi_{-v}\right) e^{-t \Delta_{v}^{p}}\right) .
$$

Now, from [GP 1], p. 676, we have

$$
\gamma_{\beta v}=\exp \int_{0}^{\beta}\left(\Phi_{b v}^{*} f_{v}\right) d b .
$$

Differentiating at $\beta=1$ and replacing $v$ by $-v$, we obtain

$$
\Phi_{v}^{*} \psi_{-v}=f_{-v}=-f_{v}
$$

The result now follows.

Combining the previous lemmas we at last obtain a formula for the variation of the determinant ratio (3.1).

Theorem 3.8. Let $\left\{X_{i}\right\}$ be a basis of $\operatorname{ker}\left(\Delta_{v}^{1}\right)$ which is $L^{2}$-orthonormal with respect to g. Let $a_{2}^{1}, a_{2}^{0}$ be the second-order heat kernel densities for the operators $\Delta_{v}^{1}, \Delta_{v}^{0}$ respectively (see (3.4)). Then

$$
\delta \log \sqrt{\frac{\operatorname{det} \Delta_{A}^{0}}{\operatorname{det} \Delta_{A}^{-}}}=-\frac{1}{16 \pi^{2}} \int_{M} f_{v}\left(\operatorname{tr}\left(\imath^{*} a_{2}^{1}\right)-2 \operatorname{tr}\left(\imath^{*} a_{2}^{0}\right)\right)+\int_{M} f_{v} \sum\left|X_{i}\right|^{2} .
$$

Both integrals in the last equation are independent of $\xi$; in particular, the $\xi$-dependence of $\left\{X_{i}\right\}$ and the inner product cancel.

Proof. We must evaluate the right-hand side of (3.19). For $\operatorname{Re}(s) \gg 0$ the previous two lemmas give

$$
\frac{1}{2}\left(\delta B_{-}(s)-\delta B_{0}(s)\right)=\int_{0}^{\infty} t^{s}\left[\frac{d}{d t} \operatorname{Tr}\left(f_{v}\left(e^{-t \Delta_{v}^{1}}-2 e^{-2 t \Delta_{v}^{0}}\right)\right)\right] d t .
$$

As in the proof of Lemma 3.7,

$$
\operatorname{Tr}\left(f_{v} e^{-t \Delta_{v}^{p}}\right)=\int_{M} f_{v} \operatorname{tr}\left(\imath K_{p}(t)\right) .
$$

Therefore

$$
\frac{d}{d t} \operatorname{Tr}\left(f_{v} e^{-t \Delta_{v}^{p}}\right)=\int_{M} f_{v} \frac{d}{d t} \operatorname{tr} \imath^{*} K_{p}(t)=\int_{M} f_{v} \frac{d}{d t} l^{*} K_{p}^{+}(t),
$$


since $K_{p}-K_{p}^{+}$is independent of $t$ (see (3.7)). Therefore the right-hand side of (3.24) equals

$$
\int_{0}^{\infty} t^{s} \frac{d}{d t}\left[\int_{M} f_{v}(x) \operatorname{tr}\left(\imath^{*} K_{1}^{+}(t)-2 \imath^{*} K_{0}(t)\right)\right] d t .
$$

Integrating by parts, we may write this as

$$
\lim _{\varepsilon \rightarrow 0}\left\{\left.t^{s} \int_{M} f_{v} \operatorname{tr}\left(\imath^{*} K_{1}^{+}-2 \imath^{*} K_{0}\right)(t)\right|_{\varepsilon} ^{1 / \varepsilon}-s \int_{\varepsilon}^{1 / \varepsilon} t^{s-1} \int_{M} f_{v} \operatorname{tr}\left(\imath^{*} K_{1}^{+}-2 K_{0}\right)(t) d t\right\} .
$$

Now as $t \rightarrow 0$ we have $\left|\operatorname{tr} \imath^{*} K_{1}^{+}(t)\right|+\left|\operatorname{tr} \imath^{*} K_{0}(t)\right| \leqq c t^{-2}$. Hence, since $\left|f_{v}\right| \leqq$ const,

$$
\left|\varepsilon^{s} \int_{M} f_{v} \operatorname{tr}\left(\imath^{*} K_{1}^{+}-2 K_{0}\right)(\varepsilon)\right| \leqq c \varepsilon^{\mathrm{Re}(s)-2} .
$$

As $t \rightarrow \infty$ then, just as in the proof of Lemma A.1,

$$
\left|\operatorname{tr} \imath^{*} K_{1}^{+}(t)\right|+\left|\operatorname{tr} \imath^{*} K_{0}(t)\right| \leqq c e^{-\lambda t}
$$

for some $\lambda>0$, so

$$
\left|\varepsilon^{-s} \int_{M} f_{v} \operatorname{tr}\left(\imath^{*}\left(K_{1}^{+}-2 K_{0}\right)\right)\left(\varepsilon^{-1}\right)\right| \leqq c \varepsilon^{-\operatorname{Re}(s)} e^{-\lambda / \varepsilon} .
$$

Hence, for $\operatorname{Re}(s) \gg 0$, the first half of (3.25) vanishes, leaving

$$
-s \int_{0}^{\infty} t^{s-1} \int_{M} f_{v} \operatorname{tr} \imath^{*}\left(K_{1}^{+}-2 K_{0}\right)(t) d t
$$

As in Lemma A.1, this expression continues analytically to a neighborhood of zero, and

$$
\begin{aligned}
\delta \log \sqrt{\frac{\operatorname{det} \Delta_{A}^{0}}{\operatorname{det} \Delta_{A}^{-}}}=\frac{1}{2}\left(\delta B_{-}(0)-\delta B_{0}(0)\right) \\
=-\operatorname{Res}_{s=0}\left\{\text { analytic continuation of } \int_{0}^{\infty} t^{s-1}\left[\int_{M} f_{v} \operatorname{tr} \imath^{*}\left(K_{1}^{+}-2 K_{0}\right)(t)\right] d t\right\} .
\end{aligned}
$$

The residue is evaluated by substituting the parametrix (3.5) into (3.26) and integrating. First, as in the proof of Lemma A.1 (i), the residue is unchanged if we replace $\int_{0}^{\infty}$ by $\int_{0}^{1}$ above. Writing $\imath^{*} K_{1}^{+}(t)=\imath^{*} K_{1}(t)-\sum\left|X_{i}\right|^{2}$ we then need to compute the residue of the continuation of

$$
-\left\{\int_{0}^{1} t^{s-1}\left[\int_{M} f_{v} \operatorname{tr}\left(\imath^{*} K_{1}-2 \imath^{*} K_{0}\right)(t)\right] d t-\int_{0}^{1} t^{s-1}\left[\int_{M} f_{v} \sum\left|X_{i}\right|^{2} d x\right] d t\right\} .
$$

The argument of Lemma 3.4 shows that the first integral gives a residue of

$$
\frac{1}{16 \pi^{2}} \int_{M} f_{v}\left(\operatorname{tr}\left(\imath^{*} a_{2}^{1}\right)-2 \operatorname{tr}\left(\imath^{*} a_{2}^{0}\right) .\right.
$$

Using $\int_{0}^{1} t^{s-1} d t=1 / s$, the result now follows. 


\section{Heat Kernel Coefficients}

The analytic results of the previous sections reduce the task of computing determinants to the more algebraic problem of finding the diagonal heat kernel coefficients $\operatorname{tr} l^{*} a_{2}$ for various operators. In this section we will calculate these coefficients. Such calculations appear, in several contexts, in the physics literature (cf. [RoSc]).

Suppose $E$ is a hermitian vector bundle with a metric-compatible connection $\nabla$, and suppose $V \rightarrow M$ is a vector bundle associated to the frame bundle of $M^{4}$. Let $\nabla=\nabla^{\text {Levi-Civita }} \otimes \mathrm{Id}_{E}+\mathrm{Id}_{V} \otimes \nabla$ be the induced connection on $V \otimes E$, and consider the operator

$$
D=\left|\nabla^{*}\right| \nabla+B
$$

where $B$ is an operator of order zero. The corresponding heat kernel $K_{D}$ has a parametrix whose second coefficient $a_{2}(x, x)$ is a section of $\operatorname{End}(V \otimes E) \otimes \Lambda^{4} T^{*} M$. By the general formula of Gilkey $[\mathrm{G}]$ its trace is

$$
\begin{aligned}
\operatorname{tr} a_{2}(x, x)= & {\left[(\operatorname{rank}(V \otimes E)) h(x)+\frac{1}{12} \operatorname{tr}\left(K_{i j} K^{i j}\right)+\frac{1}{6} \Delta(\operatorname{tr}(B))\right.} \\
& \left.-\frac{R}{6} \operatorname{tr}(B)+\frac{1}{2} \operatorname{tr}\left(B^{2}\right)\right] d M,
\end{aligned}
$$

where $K_{i j}$ is the curvature of the bundle $V \otimes E, R$ is the scalar curvature of $M$, and

$$
h(x)=-\frac{\Delta R}{30}+\frac{R^{2}}{72}-\frac{1}{180} R_{i j} R^{i j}+\frac{1}{180} R_{i j k l} R^{i j k l}
$$

is a function depending only on the geometry of $M$. (Here $R_{i j}$ are the components of the Ricci tensor.) Our primary interest is in the case of the standard sphere $M=S^{4}$, on which $h$ is an easily computed constant. However, in the next lemma we will apply (4.2-3) on a general 4-manifold $M$. We assume that $E$ is associated by a representation $\varrho$ to a principal bundle $P$ with simple structure group $G$ and that the connection $\nabla=\nabla^{A}$ is induced by a connection $A$ on $P$, whose curvature we denote by $F_{A}$. As in Sect. 1, we take the (squared) norm on the Lie algebra $g$ to be $-\left(4 \xi^{2}\right)^{-1}$ times the Killing form. We use $c_{e}$ to denote the Casimir constant of the representation $\varrho$, the ratio $\operatorname{tr}\left(\varrho(X)^{2}\right) / \operatorname{tr}\left((\operatorname{ad} X)^{2}\right)$ for any nonzero $X \in \mathfrak{g}$.

Lemma 4.1. Let $\Delta_{2}^{0}, \Delta_{A}^{1}$, and $\Delta_{A}^{-}$be the Laplacians defined in (1.3) (with $\operatorname{Ad} P$ replaced by $E$ ), and let $a_{2}^{0}, a_{2}^{1}$, and $a_{2}^{-}$denote the corresponding second heat kernel coefficients. Then we have the following pointwise formulas:

$$
\begin{gathered}
\operatorname{tr}\left(\imath^{*} a_{2}^{0}\right)=\left[(\operatorname{dim} \varrho) h-\frac{2}{3} \xi^{2} c_{\varrho}\left|F_{A}\right|_{\xi}^{2}\right] d M, \\
\operatorname{tr}\left(\imath^{*} a_{2}^{1}\right)=\left[(\operatorname{dim} \varrho) l+\frac{8}{3} \xi^{2} c_{\varrho}\left(5\left|F_{A}^{+}\right|_{\xi}^{2}-\left|F_{A}^{-}\right|_{\xi}^{2}\right)\right] d M,
\end{gathered}
$$

where $l(x)=\frac{\Delta R}{30}-\frac{R^{2}}{9}+\frac{43}{90} R_{i j} R^{i j}-\frac{11}{180} R_{i j k l} R^{i j k l}$.

(Note that the $\xi$-dependence cancels in these expressions.) 
Proof. (a) Apply (4.2) with $V=$ trivial line bundle, and $D=\Delta_{A}^{0}$. In this case, $\mid \nabla=\nabla$, $B=0$, and $K_{i j}=\varrho\left(F_{i j}\right)$, where $F=F_{A}$. Since

$$
\operatorname{tr}\left(\varrho\left(F_{i j}\right) \varrho\left(F^{i j}\right)\right)=c_{\varrho} \operatorname{tr}\left(\operatorname{ad}\left(F_{i j}\right) \operatorname{ad}\left(F^{i j}\right)\right)=-8 \xi^{2} c_{\varrho}\left|F_{A}\right|_{\xi}^{2},
$$

the result follows.

(b) It is convenient here to introduce "raising" and "lowering" operators on the exterior algebra $\Lambda^{*}\left(T^{*} M\right)$ at a point. Let $\left\{e_{i}\right\}$ be a local orthonormal basis of $T M$ and let $\left\{\theta^{i}\right\}$ be the dual basis of $T^{*} M$. We can then define operators $a_{i}^{*}(\cdot)=\theta^{i} \wedge(\cdot)$, which raise degree by 1 , and $\left.a_{i}(\cdot)=e_{i}\right\lrcorner(\cdot)$, which lower degree by 1 . If we restrict the operator $a_{i}^{*} a_{j}$ to 1 -forms, we have

$$
\operatorname{tr}_{1}\left(a_{i}^{*} a_{j}\right) \stackrel{\text { def }}{=} \operatorname{tr}\left(\left.a_{i}^{*} a_{j}\right|_{\Lambda^{1}}\right)=\sum_{k}\left(a_{i}^{*} a_{j} \theta^{k}, \theta^{k}\right)=\delta_{i j}
$$

Now apply (4.2) with $V=T^{*} M$ and $D=\Delta_{A}^{1}=d_{A} d_{A}^{*}+2\left(d_{A}^{-}\right)^{*} d_{A}^{-}$. By the Weitzenböck formula ([FU] Eq. 6.25) we have (4.1) with

$$
B=R_{i j} a_{i}^{*} a_{j} \otimes 1-2 a_{i}^{*} a_{j} \otimes \varrho\left(F_{i j}^{+}\right) .
$$

Moreover $K_{i j}=(\operatorname{Riem})_{i j} \otimes 1+1 \otimes \varrho\left(F_{i j}^{+}\right)$, where $(\text {Riem })_{i j}$ is the endomorphism with matrix $R_{l i j}^{k}$. The lemma then follows directly from (4.2) and the following observations:

(i) Since $F_{i j}=-F_{j i}$, (4.5) gives $\operatorname{tr}(B)=R \operatorname{dim} \varrho$.

(ii) For each $i, j$, (Riem) $)_{i j}$ is traceless, and $\left.\operatorname{tr}_{1}(\operatorname{Riem})_{i j}^{2}\right)=R_{l i j}^{k} R_{k i j}^{l}$. Hence

$$
\operatorname{tr}\left(K_{i j} K^{i j}\right)=-R_{i j k l} R^{i j k l}(\operatorname{dim} \varrho)-32 \xi^{2} c_{\varrho}\left|F^{+}\right|_{\xi}^{2}
$$

using (4.4).

(iii) Using $\operatorname{tr}_{1}\left(a_{i}^{*} a_{j} a_{k}^{*} a_{l}\right)=\sum_{m}\left(\theta^{m}, a_{i}^{*} a_{j} a_{k}^{*} a_{l} \theta^{m}\right)=\delta_{j k} \delta_{i l}$, together with (4.4), we have

$$
\begin{aligned}
\operatorname{tr}\left(B^{2}\right)= & \operatorname{tr}_{1}\left(\left(R_{i j} a_{i}^{*} a_{j}\right)^{2}\right)(\operatorname{dim} \varrho)-4 R_{k l} \operatorname{tr}\left(\varrho\left(F_{k l}^{+}\right)\right)+4 \operatorname{tr}\left(\left(a_{i}^{*} a_{j} \otimes \varrho\left(F_{i j}^{+}\right)\right)^{2}\right) \\
& =R_{i j} R^{i j} \operatorname{dim} \varrho+32 \xi^{2} c_{\varrho}\left|F^{+}\right|_{\xi}^{2} . \quad \square
\end{aligned}
$$

We now specialize these results to self-dual connections over $S^{4}$.

Corollary 4.2. If we take $A$ self-dual, $\varrho$ the adjoint representation of $S U(2)$, and $M=S^{4}$ with its standard metric, then

(a) $\operatorname{tr}\left(\imath^{*} a_{2}^{0}\right)=\left(\frac{29}{5}-\frac{2}{3} \xi^{2}\left|F_{A}\right|_{\xi}^{2}\right) d M$,

(b) $\operatorname{tr}\left(\imath^{*} a_{2}^{1}\right)=\left(-\frac{4}{5}+\frac{40}{3} \xi^{2}\left|F_{A}\right|_{\xi}^{2}\right) d M$.

Proof. The sphere has constant curvature $1\left(R_{i j k l}=g_{i k} g_{j l}-g_{i l} g_{j k}\right)$, implying $R \equiv 12$, $R_{i j} R^{i j} \equiv 36$, and

$$
R_{i j k l} R^{i j k l} \equiv 24 \equiv 2 R_{i j k l}^{+} R^{+i j k l} \equiv 2 R_{i j k l}^{-} R^{-i j k l} .
$$

Hence we find $h(x) \equiv 29 / 15$ and $l(x) \equiv-4 / 15$. We also have $\operatorname{dim} \varrho=3, c_{\varrho}=1$, and $F_{A}^{-}=0$. The results now follow from Lemma 4.1 .

We can use this result to compute the value of $\zeta(0)$ on the self-dual moduli spaces $\mathscr{M}_{k}\left(S^{4}\right)$ of $S U(2)$ connections on the standard 4-sphere. (This refines Corollary 3.3.) 
Corollary 4.3. The functions $\zeta_{\Delta}^{0}(0)$ and $\zeta_{\Delta_{A}^{1}}(0)$ are constant on $\mathscr{M}_{k}^{*}\left(S^{4}\right)$. Specifically, we have the following:

$$
\begin{aligned}
& \zeta_{\Delta_{A}^{0}}(0)=\left\{\begin{array}{lll}
-\frac{61}{30} & \text { if } & k=0 \\
\frac{29}{30}-\frac{k}{3} & \text { if } & k>0 .
\end{array}\right. \\
& \zeta_{\Delta_{A}^{1}}(0)=\left\{\begin{array}{lll}
-\frac{2}{15} & \text { if } & k=0 \\
\frac{43}{15}-\frac{4}{3} k & \text { if } & k>0
\end{array}\right. \\
& \zeta_{\Delta_{A}}(0)=\frac{19}{10}-k \quad \forall k .
\end{aligned}
$$

Proof. When $k=0$ the moduli space consists of a single point, the trivial connection $\theta$. For the $\theta$-Laplacians, the dimension of the kernel of $\Delta_{\theta}^{p}$ is simply the dimension of $s u(2)$ times the dimension of the kernel of the Laplacian on scalar-valued $p$-forms on $S^{4}$, and hence $\operatorname{dim} \operatorname{ker} \Delta_{\theta}^{0}=3$ and $\operatorname{dim} \operatorname{ker} \Delta_{\theta}^{1}=0$. For a connection $A$ in the smooth part of $\mathscr{M}_{k}\left(S^{4}\right)$ with $k>0$ we have $\operatorname{dim} \operatorname{ker} \Delta_{A}^{0}=0$ and, by the index theorem (cf. [AHS]), $\operatorname{dim} \operatorname{ker} \Delta_{A}^{1}=8 k-3$. The above results then follow by substituting these dimensions into (3.12), applying Corollary 4.2, and noting that $\operatorname{Vol}\left(S^{4}\right)$ $=8 \pi^{2} / 3$.

Remarks. (i) The corresponding conclusion holds for $k<0$; one need only replace $k$ with $|k|, \Delta_{A}^{-}$with $\Delta_{A}^{+}$, and $\Delta_{A}^{1}$ with $d_{A} d_{A}^{*}+2\left(d_{A}^{+}\right) *\left(d_{A}^{+}\right)$.

(ii) The values of $\zeta(0)$ in Corollary 4.3 are independent of $\xi$, and are invariant under constant rescalings of the metric on $S^{4}$.

Finally, we apply Corollary 4.3 to describe how the semiclassical measure (2.17) and the determinant ratio $C_{0}$ in (2.18) depend on $\xi$.

Corollary 4.4. The constant $C_{0}(\xi)$ in (2.18) satisfies

$$
C_{0}(\xi)=\left(2 \pi \xi^{2}\right)^{\frac{19}{20}} \quad C_{0}(1)
$$

and the semiclassical measure on $\mathscr{M}_{k}\left(S^{4}\right), k \neq 0$, satisfies

$$
d v_{\xi}=\left(2 \pi \xi^{2}\right)^{\frac{19}{20}-\frac{|k|}{2}} d v_{1} \text {. }
$$

Proof. The Laplacian $\Delta_{\xi}^{-}$is related to $\Delta_{A}^{-}$by (2.9). Applying Lemma $3.1 \mathrm{~b}$ to the definition (2.17), we have

$$
d v_{\xi}=\sqrt{\frac{\operatorname{det} \Delta^{0}}{\operatorname{det} \Delta_{\xi}^{-}}} d \mathscr{M}=\left(2 \pi \xi^{2}\right)^{\frac{1}{2} \zeta-(0)} \sqrt{\frac{\operatorname{det} \Delta_{A}^{0}}{\operatorname{det} \Delta_{A}^{-}}} d \mathscr{M} .
$$

The result then follows from Corollary 4.3.

\section{The Semiclassical Measure}

Having calculated the values for the heat kernel coefficients $a_{2}$ we can now evaluate the right-hand side of (3.23). This leads to the first main result of this paper: the semi classical measure of $\mathscr{M}_{1}$ is finite.

To start, we will take the coefficient $\xi$ introduced in Sect. 1 to have its standard value 1. This simplifies the calculations and maintains consistency with [GP 1], 
whose results we will use. In the end we can recover the $\xi$-dependence from Lemma $3.1 \mathrm{~b}$ (cf. Corollaries 4.4 and 7.2).

Lemma 3.2b of [GP 1] asserts that $\left|F_{v}\right|^{2}=3 \gamma_{v}^{4}$. Combining this with the result of Corollary 4.2, we have $\operatorname{tr} \imath^{*} a_{2}^{1}-2 \operatorname{tr} \imath^{*} a_{2}^{0}=44 \gamma_{v}^{4}+$ constant. Since $\int_{S^{4}} f_{v}=0$, it follows that

$$
\int_{S^{4}} f_{v}\left(\operatorname{tr} \imath^{*} a_{2}^{1}-2 \operatorname{tr} \imath^{*} a_{2}^{0}\right)=44 \int_{S^{4}} f_{v} \gamma_{v}^{4} .
$$

We will evaluate this integral shortly, expressing it in terms of integrals

$$
I_{p}(v)=\frac{1}{4 \pi^{2}} \int_{S^{4}} f_{\hat{v}}^{p} \gamma_{v}^{4}, \quad \text { where } \hat{v}=v /|v| .
$$

To obtain a final answer we will therefore need the following lemma

Lemma 5.1. Let $r=|v|, \alpha=\cosh r, \beta=\sinh r$. Then

$$
I_{1}(v)=\frac{2}{3} \alpha \beta^{-1}-\alpha \beta^{-3}+r \beta^{-4} \stackrel{\text { def }}{=} I_{1}(r),
$$

and

$$
I_{3}(v)=\frac{2}{3} \alpha \beta^{-1}-\frac{7}{3} \alpha \beta^{-3}-10 \alpha \beta^{-5}+9 r \beta^{-4}+10 r \beta^{-6} \stackrel{\text { def }}{=} I_{3}(r) .
$$

Proof. One computes as in the appendix of [GP 1]. We leave the details to the reader.

For the rest of this section we use the letters $\alpha, \beta$, and $r$ have the same meaning as in Lemma 5.1. We will also make use of the functions

and

$$
A(r)=\beta^{-2}+3 \beta^{-4}-3 r \alpha \beta^{-5}
$$

$$
B(r)=3 \alpha^{-1} \beta I_{1} .
$$

The importance of these functions lies in the fact, shown in [GP 1, Sect. 6], that the metric on $\mathscr{M}_{1}$ takes the form

$$
\mathbf{g}=8 \pi^{2} A(r) d r^{2} \oplus 2 \pi^{2} B(r) g,
$$

where $g$ is, in Sect. 3, is the standard metric on $S^{4}$.

We now turn to the evaluation of $\int f_{v} \sum_{i=1}^{5}\left|X_{i}\right|^{2}$. From Proposition 4.3 of [GP 1] we know that $\operatorname{ker}\left(\Delta_{v}^{1}\right)=\left\{l_{Y_{w}} F^{v} \mid w \in \mathbf{R}^{5}\right\}$, where $Y_{w}=\operatorname{grad}\left(f_{w}\right)$. The proposition below follows from the results of [GP 1, Sect. 6].

Proposition 5.2. Let $v \neq 0$ and let $\left\{w_{i}\right\}_{1}^{5}$ be an orthonormal basis of $\mathbf{R}^{5}$ with

$$
w_{1}=v /|v| \stackrel{\text { def }}{=} \hat{v} \text {. }
$$

Write $Y_{i}=Y_{w_{i}}$. Then

(a) Pointwise, $\left|l_{Y_{w}} F^{v}\right|^{2}=\frac{3}{2} \gamma_{v}^{4}\left(|w|^{2}-f_{w}^{2}\right)$.

(b) $\left\{l_{Y_{i}} F^{v}\right\}$ is an (un-normalized) orthogonal basis of $\operatorname{ker}\left(\Delta_{v}^{1}\right)$.

(c) $\left\|l_{Y_{1}} F^{v}\right\|^{2}=8 \pi^{2} A(r) \stackrel{\text { def }}{=} \varrho^{2}$.

(d) $\left\|l_{Y_{i}} F^{v}\right\|^{2}=2 \pi^{2}(2-A(r))=6 \pi^{2} \alpha \beta^{-1} I_{1} \stackrel{\text { def }}{=} \sigma^{2}, i \neq 1$.

These facts quickly lead to the following corollary. 
Corollary 5.3. At each $A_{v} \in \mathscr{M}_{1}$,

$$
\delta \log \sqrt{\frac{\operatorname{det} \Delta_{A}^{0}}{\operatorname{det} \Delta_{A}^{-}}}=r\left\{-11 I_{1}+\frac{3}{4} A(r)^{-1}\left(I_{1}-I_{3}\right)+\alpha^{-1} \beta\left(3+I_{3} / I_{1}\right)\right\} .
$$

Proof. Parts (b), (c), and (d) of Proposition 5.2 imply that

$$
\sum_{i=1}^{5}\left|X_{i}\right|^{2}=\varrho^{-2}\left|l_{Y_{1}} F^{v}\right|^{2}+\sigma^{-2} \sum_{i=2}^{5}\left|l_{Y_{i}} F^{v}\right|^{2} .
$$

But, using part (a) and the identity $\sum_{i=1}^{5} f_{i}^{2}=1$, we find

We also have

$$
\sum_{i=2}^{5}\left|l_{Y_{i}} F^{v}\right|^{2}=\frac{3}{2} \gamma_{v}^{4}\left(3+f_{\hat{v}}^{2}\right) \text {. }
$$

$$
\left|l_{Y_{1}} F^{v}\right|^{2}=\frac{3}{2} \gamma_{v}^{4}\left(1-f_{\hat{v}}^{2}\right)
$$

so we obtain

$$
\int_{S^{4}} f_{v} \sum\left|X_{i}\right|^{2}=4 \pi^{2}|v|\left(\frac{3}{2} \varrho^{-2}\left[I_{1}(v)-I_{3}(v)\right]+\frac{3}{2} \sigma^{-2}\left[3 I_{1}(v)+I_{3}(v)\right]\right) .
$$

Substituting (5.1) and (5.6) into (3.23), the result follows.

To proceed further, it is useful to re-express the volume forms (classical and semiclassical) in terms of the scale function $\lambda$ on $\mathscr{M}_{1}$ defined by (1.7). On $S^{4}$ the function $\left|F_{A_{0}}\right|$ is constant, so $\lambda\left(A_{0}\right)=\frac{1}{2} \operatorname{diam}\left(S^{4}\right)=\frac{\pi}{2}$. Since the Yang-Mills density is conformally invariant and the flows $\Phi_{v}$ used to parametrize $\mathscr{M}_{1}$ are conformal (see Sect. 3), $\lambda\left(A_{v}\right)$ is simply the spherical distance from the $\Phi_{v}$-image of the equator perpendicular (in $\mathbf{R}^{5}$ ) to $v$ to the closer of the poles $\pm \hat{v}$. For a point $x$ on the equator one has $\Phi_{v}(x)=-(\tanh r) \hat{v}+(\operatorname{sech} r) x$, and hence $\lambda\left(A_{v}\right)=\tan ^{-1}(\operatorname{sech} r / \tanh r)$. Thus we obtain the following relations.

$$
\begin{aligned}
\lambda & =\tan ^{-1}(\operatorname{csch} r), \\
r & =\log \cot (\lambda / 2) .
\end{aligned}
$$

Asymptotically we therefore have

$$
\begin{gathered}
\lambda \sim 2 e^{-r} \quad \text { as } \quad r \rightarrow \infty, \\
r \sim-\log \lambda \text { as } \lambda \rightarrow 0 .
\end{gathered}
$$

We can now compute the asymptotic behavior of (3.1) near the boundary of the moduli space. From Lemma 5.1 one finds that as $r \rightarrow \infty, A(r)=4 e^{-2 r}\left(1+O\left(r e^{-2 r}\right)\right)$, $I_{1}=\frac{2}{3}\left(1+O\left(e^{-2 r}\right)\right)=I_{3}$, and

$$
I_{1}-I_{3}=\frac{16}{3} e^{-2 r}\left(1+O\left(e^{-2 r}\right)\right) .
$$

Comparing with (5.2), this implies

$$
\delta \log \sqrt{\frac{\operatorname{det} \Delta_{A}^{0}}{\operatorname{det} \Delta_{A}^{-}}}=-\frac{7}{3} r+O\left(r^{2} e^{-2 r}\right) .
$$

When we re-express this relation in terms of $\lambda$ we obtain the finiteness statement of Theorem 0.1 of the introduction. 
Corollary 5.4. The semiclassical measure of $\mathscr{M}_{1}$ is finite. In fact, as $\lambda \rightarrow 0$, we have

$$
\sqrt{\frac{\operatorname{det} \Delta_{A}^{0}}{\operatorname{det} \Delta_{A}^{-}}} \sim C \lambda^{7 / 3}
$$

for some constant $C$.

Proof. In the usual coordinates on $\mathbf{R}^{5}$, the variation vector field $\delta$ we have introduced is simply $r \frac{\partial}{\partial r}$. Thus (5.5) implies that

$$
\frac{d}{d r}\left(\log \sqrt{\frac{\operatorname{det} \Delta_{A}^{0}}{\operatorname{det} \Delta_{A}^{-}}}\right)=-\frac{7}{3}+O\left(r e^{-2 r}\right)
$$

and hence

$$
\sqrt{\frac{\operatorname{det} \Delta_{A}^{0}}{\operatorname{det} \Delta_{A}^{-}}} \sim \text { const } \cdot e^{-\frac{7}{3} r}
$$

Using (5.10), (5.12) follows, and we see that the ratio of the semiclassical volume form to the classical one is bounded. Since the volume of $\mathscr{M}_{1}$ is finite ([GP 1 , Corollary B]) the semiclassical measure is therefore finite as well.

In principle Corollary 5.3 allows us to compute the determinant ratio (3.1) everywhere on $\mathscr{M}_{1}$ exactly, up to the multiplicative factor $C_{1}=\sqrt{\frac{\operatorname{det} \Delta_{A_{0}}^{0}}{\operatorname{det} \Delta_{A_{0}}^{-}}}$which we will compute in the next section. Because of the complexity of the right-hand side of (5.5), however, one should not expect the determinant ratio to be an elementary function of $r$ or $\lambda$. For now we will simply lay out the formulas needed for this computation, deferring the actual calculations until Sect. 6. It is simpler to work in terms of $\lambda$ then in terms of $r$, so we write

$$
\sqrt{\frac{\operatorname{det} \Delta_{A}^{0}}{\operatorname{det} \Delta_{A}^{-}}}=C_{1} w(\lambda)
$$

where the function $w(\lambda)$ is determined by integrating (5.5) with the normalization $w(\pi / 2)=1$. Specifically, using $d / d \lambda=-\csc \lambda d / d r, \alpha \beta^{-1}=\sec \lambda$, and $\beta^{-1}=\tan \lambda$, (5.5) becomes

$$
\frac{d}{d \lambda} \log w=-\csc \lambda\left\{-11 I_{1}(\lambda)+\frac{3}{4 A(\lambda)}\left(I_{1}(\lambda)-I_{3}(\lambda)\right)+\cos \lambda\left(3+\frac{I_{3}(\lambda)}{I_{1}(\lambda)}\right)\right\},
$$

where

$$
\begin{gathered}
I_{1}(\lambda)=\frac{2}{3} \sec \lambda-\sec \lambda \tan ^{2} \lambda+r(\lambda) \tan ^{4} \lambda, \\
I_{3}(\lambda)=\sec \lambda\left(\frac{2}{3}-\frac{7}{3} \tan ^{2} \lambda-10 \tan ^{4} \lambda\right)+r(\lambda)\left(9 \tan ^{4} \lambda+10 \tan ^{6} \lambda\right), \\
A(\lambda)=\tan ^{2} \lambda\left(1+3 \tan ^{2} \lambda-3 r(\lambda) \sec \lambda \tan ^{2} \lambda\right),
\end{gathered}
$$

and where $r(\lambda)$ is given by (5.8). To compute expectations using these formulas we will also need to have the volume form $d \mathscr{M}_{1}$ expressed in terms of $\lambda$. If we rewrite the metric (5.4) in terms of $\lambda$ we find

$$
\mathbf{g}=4 \pi^{2}\left(2 f(\lambda) d \lambda^{2} \oplus h(\lambda) g\right)
$$


where $g$ is the standard metric on $S^{4}$,

and

$$
f(\lambda)=\csc ^{2} \lambda A(\lambda)=\sec ^{2} \lambda\left(1+3 \tan ^{2} \lambda-3 r(\lambda) \sec \lambda \tan ^{2} \lambda\right)
$$

$$
h(\lambda)=\frac{1}{2} B(\lambda)=1-\frac{3}{2} \tan ^{2} \lambda+\frac{3}{2} r(\lambda) \cos \lambda \tan ^{4} \lambda
$$

(cf. [DMM, Sect. 3] and [GP 2, Theorem II]). In terms of these functions, the Riemannian volume form on $\mathscr{M}_{1}$ is then given by

$$
d \mathscr{M}_{1}=(2 \pi)^{5} \sqrt{2 f(\lambda)} h(\lambda)^{2} d \lambda \wedge d M,
$$

and the semiclassical volume form by

$$
d v=C_{1} w(\lambda) d \mathscr{M}_{1}
$$

Equations (5.13)-(5.18) give an explicit, albeit complicated, formula for the semiclassical measure on the moduli space $\mathscr{M}_{1}$. With this formula in hand we can now return to the original problem of calculating semiclassical expectations.

\section{Semiclassical Expectations on $\mathscr{M}_{1}$}

Let $\Phi$ be any observable that vanishes on $\mathscr{B}_{0}$. From (2.18) and Corollary 4.4, its semiclassical expectation is given by

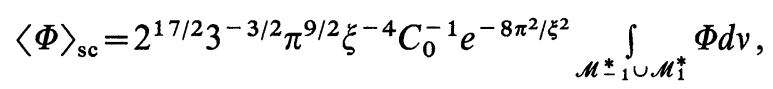

where the determinant ratios defining $C_{0}$ and $d v$ are taken with $\xi=1$ (and provided the integral is not 0 ). When $M=S^{4}$ we have $\mathscr{M}_{ \pm 1}^{*}=\mathscr{M}_{ \pm 1}$, and the antipodal map on $S^{4}$ induces an isometry $\alpha: \mathscr{M}_{1} \leftrightarrow \mathscr{M}_{-1}$ that preserves the semiclassical measure. We can then write $\Phi$ as the sum $\Phi^{\text {ev }}+\Phi^{\text {odd }}$ of functions that are even and odd with respect to $\alpha$. The integral (6.1) of $\Phi^{\text {odd }}$ vanishes, and the integrals of $\Phi_{\text {ev }}$ over the $k= \pm 1$ moduli spaces are equal. As in Sect. 5 , on $\mathscr{M}_{1}$ we can write

$$
\sqrt{\operatorname{det}\left(\Delta^{0}\right) / \operatorname{det}\left(\Delta^{-}\right)}=C_{1} w(\lambda)
$$

for some constant $C_{1}$. We then have

$$
\langle\Phi\rangle_{\mathrm{sc}}=2^{19 / 2} 3^{-3 / 2} \pi^{9 / 2} \frac{C_{1}}{C_{0}} e^{-8 \pi^{2} / \xi^{2}} \int_{\mathscr{M}_{1}} \Phi^{\mathrm{ev}} w(\lambda) d \mathscr{M} .
$$

From the previous section we know $d \mathscr{M}$ and the logarithmic derivative of $w$, so in principle we can compute the measure $w(\lambda) d \mathscr{M}$. The remaining unknown quantity in (6.3) is therefore $C_{1} / C_{0}$. This quotient of determinant ratios is not expressible in terms of local data, but there is sufficient cancellation among the $\zeta$-functions and enough symmetry that we can still calculate it. To get started, let us write $D_{0}^{p}=\Delta_{\theta}^{p}$ and $D_{1}^{p}=\Delta_{A_{0}}^{p}$, where we let $p$ stand for 0,1 , or - . (Here $\theta$ is the trivial flat $k=0$ connection and $A_{0}$ is the standard $k=1$ instanton; see Sect. 3.) We will abbreviate $\zeta_{D_{k}^{p}}(s)$ as $\zeta_{p, k}(s)$. Since $\zeta^{\prime}(0)=-\log d e t$, we can use the $\zeta$-function combination

$$
z(s)=\frac{1}{2}\left[\zeta_{0,1}(s)-\zeta_{-, 1}(s)-\zeta_{0,0}(s)+\zeta_{-, 0}(s)\right]
$$

to compute $C_{1} / C_{0}$. By Corollary 3.3 this can be rewritten as

$$
z(s)=\frac{1}{2}\left\{2\left[\zeta_{0,1}(s)-\zeta_{0,0}(s)\right]-\left[\zeta_{1,1}(s)-\zeta_{1,0}(s)\right]\right\} .
$$


Using representation theory one can determine all of the eigenvalues of the operators $D_{k}^{p}$ above; see [BP]. The number $\log C_{1} / C_{0}=-z^{\prime}(0)$ can then be computed exactly in terms of the ordinary Riemann zeta function $\zeta_{R}(s)$, as has been done by several physicists ([H], [CVDN]). The result is

$$
\begin{aligned}
C_{1} / C_{0} & =2 \cdot 3^{3 / 2} \cdot 5^{5 / 2} \cdot \exp \left(8 \zeta_{R}^{\prime}(-1)-5\right) \\
& \approx 1.042 .
\end{aligned}
$$

When the constant (6.5) is put into (6.3), we obtain a complete and explicit formula for the semiclassical expectation of observables $\Phi$ that vanish on $\mathscr{A}_{0}$. This is the formula referred to in Theorem (0.1). We can now apply this formula to calculate the expectations of the observables $|k|$ and $\lambda$ defined in Sect. 2, provided $M$ is the standard 4-sphere and $G=S U(2)$.

In the coordinate system discussed at the end of Sect. $5 \mathscr{M}_{1}$ is identified with the ball of radius $\pi / 2$ in $\mathbf{R}^{5}$, where $\lambda$ is the radial parameter and where the measure $w(\lambda) d \mathscr{M}$ is rotationally symmetric. The observable $|k|$ is identically 1 as a function on $\mathscr{M}_{1}$, so both $|k|$ and $\lambda$ are rotationally symmetric functions; they are in fact the functions $\lambda^{p}$ for $p=0,1$. Substituting (5.18) and (5.17) into (6.3) and integrating over $S^{4}$ gives

$$
\left\langle\lambda^{p}\right\rangle_{\mathrm{sc}}=C_{2} \frac{C_{1}}{C_{0}} \xi^{-4} e^{-8 \pi^{2} / \xi^{2}} \int_{0}^{\frac{\pi}{2}} \lambda^{p} f^{1 / 2}(\lambda) h^{2}(\lambda) w(\lambda) d \lambda,
$$

where

$$
C_{2}=2^{18} 3^{-5 / 2} \pi^{23 / 2}
$$

These integrals can be evaluated numerically. This computation is not entirely straightforward, but the difficulties in the numerical analysis are not relevant to our purposes here. Hence we will simply record the results, which are

$$
\int_{0}^{\frac{\pi}{2}} f^{1 / 2}(\lambda) h^{2}(\lambda) w(\lambda) d \lambda \approx 0.05718
$$

and

$$
\int_{0}^{\frac{\pi}{2}} \lambda f^{1 / 2}(\lambda) h^{2}(\lambda) w(\lambda) d \lambda \approx 0.03544 .
$$

Combining (7.1)-(7.3) and using (6.5) gives the semiclassical expectations

$$
\langle|k|\rangle_{\mathrm{sc}} \approx 0.05958 C_{2} \xi^{-4} e^{-8 \pi^{2} / \xi^{2}}
$$

and

$$
\langle\lambda\rangle_{\mathrm{sc}} \approx 0.03693 C_{2} \xi^{-4} e^{-8 \pi^{2} / \xi^{2}} .
$$

Remark. Both of these expectations are exponentially small in $\xi$ as $\xi \rightarrow 0$. However, the ratio

$$
\frac{\langle\lambda\rangle_{\mathrm{sc}}}{\langle|k|\rangle_{\mathrm{sc}}}=\frac{\int_{\mathscr{M}_{1}} \lambda d v}{\int_{\mathscr{M}_{1}} d v} \approx 0.6198
$$

is independent of $\xi$. This ratio can be interpreted as a conditional (semiclassical) expectation: the expected scale of a connection given that the connection has instanton number $k= \pm 1$. For the sake of comparison, if we replace the 
semiclassical measure $d v$ by the classical measure $d \mathscr{M}_{1}$, we obtain the "classical" conditional expectation

$$
\frac{\int_{\mathscr{M}_{1}} \lambda d \mathscr{M}_{1}}{\operatorname{Vol}\left(\mathscr{M}_{1}\right)} \approx 0.3112 .
$$

\section{Rescaling and Renormalization}

The semiclassical expectations we have been discussing depend on two choices of scale: the choice of coupling constant $\xi$ used to define the norm on $\operatorname{Ad} P$ [cf. (1.1)], and the choice of the radius $R$ of the base manifold $S^{4}$ (equivalently, the scale of the metric on $S^{4}$ ). Thus far we have taken $R=1$ (relative to the length scale $L$ described in the introduction), but it is now easy to make the dependence on $R$ explicit.

Replacing the unit sphere $S^{4}$ with the sphere $S_{R}^{4}$ of radius $R$ has the effect of replacing the standard metric $g_{0}$ with $R^{2} g_{0}$. We begin by describing how this constant rescaling of the metric affects the semiclassical expectations (2.18) for general $k$.

First note that observables generally depend on the metric. For example, the scale function $\lambda$ (Example 4 of Sect. 2) is obtained by finding the ball that contains energy $4 \pi^{2}|k|$ and measuring its radius with respect to some metric. Thus when we consider the 1-parameter family of metrics $g=R^{2} g_{0}$ each observable must be regarded as a function $\Phi=\Phi\left(A, R^{2} g_{0}\right)$ of $R$. Certain observables rescale as a simple power of $R$, i.e.

$$
\Phi\left(A, R^{2} g_{0}\right)=R^{d} \Phi\left(A, g_{0}\right)
$$

for some $d$; this number $d$ is the physical dimension of the observable. (Note here that $R$ is a dimensionless parameter, a ratio of radii.) In particular, the instanton number $|k|$ is independent of $R(d=0)$, and the scale function $\lambda$ rescales as $\lambda_{R}=R \lambda$ $(d=1)$.

Proposition 7.1. The semiclassical expectation (2.18) of an observable $\Phi$ that has dimension $d$ and that vanishes of $\mathscr{B}_{l}$ for $|l|<|k|$ rescales under constant conformal changes of metric by

$$
\langle\Phi\rangle_{\mathrm{sc}, R^{2} g_{0}}=R^{\frac{22}{3}|k|+d}\langle\Phi\rangle_{\mathrm{sc}, g_{0}}
$$

assuming, as usual, that the integrals in (2.18) don't vanish.

Proof. The Yang-Mills action $S(A)$ is conformally invariant, but the change of metric affects the other terms in (2.18) as follows:

1. The observable $\Phi$ rescales according to (7.1).

2. The volume form on $S^{4}$ becomes $R^{4} d v_{g_{0}}$ and the $L^{2}$ norm of $B \in \Omega^{1}(\operatorname{Ad} P)$ becomes

$$
\|B\|_{R^{2} g_{0}}^{2}=R^{2}\|B\|_{g_{0}}^{2} .
$$

Hence the $L^{2}$ metric $\mathbf{g}$ on $\mathscr{M}_{k}$ becomes $R^{2} \mathbf{g}$, and the volume form becomes $R^{d(k)} d \mathscr{M}$, where $d(k)$ is the dimension of $\mathscr{M}_{k}$ (which is 0 for $k=0$ and $8|k|-3$ for $k \neq 0$ ).

3. The Laplacian $\Delta^{p}$ becomes $R^{-2} \Delta^{p}$, and hence by Lemma $3.1 \mathrm{~b}$ we have

$$
\sqrt{\frac{\operatorname{det}\left(R^{-2} \Delta^{0}\right)}{\operatorname{det}\left(R^{-2} \Delta^{-}\right)}}=R^{\zeta-(0)-\zeta_{0}(0)} \sqrt{\frac{\operatorname{det}\left(\Delta^{0}\right)}{\operatorname{det}\left(\Delta^{-}\right)}} .
$$


4. From Proposition 2.1, the volume of the group $G_{c}$ of constant gauge transformations rescales by a factor of $R^{6}$. (Note also that the ratio of the volumes of the components $\mathscr{G}_{k}$ of the gauge group - which we assumed was equal to 1 in remark at the end of Sect. 2 - is formally independent of $R$.)

Combining (2) and (3) and applying Corollary 4.3, we find that the semiclassical measure $d v=\sqrt{\frac{\operatorname{det}\left(\Delta^{0}\right)}{\operatorname{det}\left(\Delta^{-}\right)}} d \mathscr{M}_{k}, k \neq 0$, becomes

$$
d v_{R}=R^{\frac{22}{3}|k|-\frac{31}{15}} d v \text {. }
$$

Similarly, from (2), (3), (4), and Corollary 4.3, we find that the effective semiclassical measure $d v=\left(\operatorname{Vol} G_{c}\right)^{-1} \sqrt{\frac{\operatorname{det}\left(\Delta^{0}\right)}{\operatorname{det}\left(\Delta^{-}\right)}}$on $\mathscr{M}_{0}$ [cf. (2.13) and Proposition 2.1c] becomes

$$
d v_{R}=R^{-31 / 15} d v .
$$

Incorporating (1), we conclude that the ratio $\left(Z_{k}(\Phi)+Z_{-k}(\Phi)\right) / Z_{0}(1)$ rescales as in (7.2).

Making these changes in (2.18) and including the $\xi$-dependence using Corollary 4.4 gives an expression for the semiclassical expectation which makes the dependence on the scale parameters $\xi$ and $R$ manifest.

Corollary 7.2. The semiclassical expectation on $S_{R}^{4}$ of an observable $\Phi$ that has dimension $d$ and that vanishes identically on $\mathscr{B}_{l}$ for $|l| \leqq|k|$ is

$$
\langle\Phi\rangle_{\mathrm{sc}, R^{2} g_{0}, \xi} \begin{cases}R^{d} \Phi(\theta) & \text { if } \quad k=0 \\ (\text { const }) C_{0}^{-1}(2 \pi)^{-|k| / 2} \xi^{-(|k|+3)} R^{\frac{22}{3}|k|+d} e^{-8 \pi^{2}|k| / \xi^{2}} \int_{\mathscr{M}_{k}} \Phi^{\mathrm{ev}} d v & \text { if } \quad k \neq 0,\end{cases}
$$

where the constant is $32 \cdot 3^{-3 / 2} \cdot(2 \pi)^{5}$.

In particular, the observables $|k|$ and $\lambda_{R}$ (the instanton number and scale size on $\left.S_{R}^{4}\right)$ vanish on $\mathscr{B}_{0}$ and, from (7.4) and (7.5), have semiclassical expectations

and

$$
\langle|k|\rangle_{\mathrm{sc}} \approx 0.05958 C_{2} \xi^{-4} R^{22 / 3} e^{-8 \pi^{2} / \xi^{2}}
$$

$$
\left\langle\lambda_{R}\right\rangle_{\mathrm{sc}} \approx 0.03693 C_{2} \xi^{-4} R^{25 / 3} e^{-8 \pi^{2} / \xi^{2}},
$$

where $C_{2}$ is the constant of (7.1).

We conclude with some speculative comments relating the above rescaling formulas to renormalization. While these comments are motivated by physical considerations, they are intended only as an example of a renormalization process. We will not directly compare them with the renormalizations described in the physics literature; such a comparison would require an examination of the Green functions and of quantum corrections beyond the semiclassical level-topics beyond the scope of this paper.

The need for renormalization can be seen physically as follows. We start with the semiclassical quantum field theory on the compact "spacetime" $S_{R}^{4}$, a theory which has well-defined semiclassical expectations. We then let $R \rightarrow \infty$, obtaining expectations on spacetimes that more and more closely resemble $\mathbf{R}^{4}$. As we do this, the expectations rescale according to (7.2). On the other hand, $\lambda_{R}$ is a local 
observable - it measures the expected size of an instanton and instantons are very localized in spacetime. Thus physical intuition suggests that the semiclassical expectation $\left\langle\lambda_{R}\right\rangle_{\mathrm{sc}}$ should be independent of $R$, at least for large $R$ (the expected size of a quantum particle should not depend on the radius of the spacetime). Renormalization provides a way of achieving this that is compatible with (7.2).

The basic idea is to make the coupling constant $\xi$ a function of $R$. We then let $R \rightarrow \infty$, simultaneously rescaling the observables [according to (7.1)] and $\xi$ in such a way that the expectations remain constant. This is accomplished by requiring that $\xi^{-4} R^{22 / 3} e^{-8 \pi^{2} / \xi^{2}}=$ constant; thus

$$
R^{22 / 3}=K_{0} \xi^{4} e^{8 \pi^{2} / \xi^{2}}
$$

for some $K_{0}$. Comparing this with (7.7), we see that the renormalized expectation of $|k|$ (and of any observable of dimension $d=0$ ) is constant. Next, because the righthand side of (7.6) depends on the dimension of the observable we also rescale the expectations of observables of dimension $d$ by the formula

$$
\langle\Phi\rangle^{\mathrm{re}}=R^{-d}\left\langle\Phi_{R^{2} g_{0}}\right\rangle ;
$$

this cancels the dependence (7.1) on $R$. With these definitions, Corollary (7.2) implies that the renormalized semiclassical expectation of an observable $\Phi$ that vanishes on $\mathscr{B}_{l}$ for $|l|<|k|$ is

$$
\langle\Phi\rangle_{\mathrm{sc}, R^{2} g_{0}}^{\mathrm{re}}=\left\{\begin{array}{lll}
\Phi(\theta) & \text { if } & k=0 \\
32 \cdot 3^{-3 / 2} C_{0}^{-1}(2 \pi)^{5-|k| / 2} K_{0}|k| \xi^{3(|k|-1)} \int_{\mathcal{M}_{k}} \Phi^{\mathrm{ev}} d v & \text { if } \quad k \neq 0 .
\end{array}\right.
$$

Thus Eqs. (7.7) and (7.8) become

$$
\langle|k|\rangle_{\mathrm{sc}} \approx 0.05958 K_{1}, \quad\langle\lambda\rangle_{\mathrm{sc}} \approx 0.03693 K_{1},
$$

where $K_{1}=C_{2} K_{0}$. Note that $K_{1}$ is dimensionless; a length scale $L$ must be put in by hand as described in the introduction.

There are several striking aspects of formula (7.11). First, the renormalized semiclassical expectations for $k=0$ and $k=1$ are independent of $R$. Thus the limit as $R \rightarrow \infty$ exists and can in fact be calculated using any value of $R$. Second, the expectation of any observable that vanishes identically on $\mathscr{B}_{0}$ can explicitly be calculated. Third, the exponential dependence on $\xi$ of the semiclassical expectations that has been with us since Eq. (2.18) has disappeared; after renormalization the moduli spaces $\mathscr{M}_{k}$ for $|k| \geqq 1$ contribute terms proportional simply to powers of $\xi$.

There is another striking feature of (7.11). Differentiating (7.9), one finds that

$$
R \frac{d \xi^{2}}{d R}=-\frac{11}{12 \pi^{2}} \xi^{4}\left(1-\frac{\xi^{2}}{4 \pi^{2}}\right)^{-1}
$$

in particular for $\xi$ is sufficiently small $R$ is a decreasing function of $\xi$.

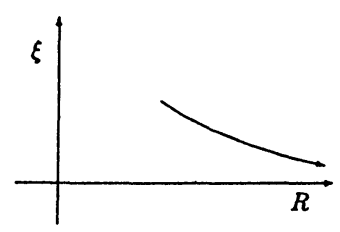


This means that as $R$ gets larger our semiclassical approximation becomes better and better. This phenomenon - that the coupling constant $\xi$ decreases at large scales - is called asymptotic freedom.

Remarks. (1) The physicists' renormalization philosophy is somewhat different from ours. Our approach is to hold the unit length scale $L$ constant while sending $R$ to infinity (and hence also the physical radius $R L$ ), simultaneously changing the coupling constant $\xi$ so that the dimensionless combination

$$
R^{22 / 3} \xi^{-4} \exp \left(-8 \pi^{2} / \xi^{2}\right)
$$

remains fixed, equalling the dimensionless constant we have named $K_{0}$. We regard $K_{0}$ as an absolute "constant of nature" and not something to be renormalized further. This view entails an implicit relation between what physicists would consider our ultraviolet and infrared cutoffs, and which they prefer to keep separate. In the physicists' approach two "mass scales," $\mu$ and $\Lambda$, are introduced. The scale $\mu$ is effectively the reciprocal of our $L$, while $\Lambda$ is related to $K_{0}, R$, and $L$; the table below provides a dictionary to pass between our notation and what is common in the physics literature.

\begin{tabular}{ll}
\hline Our notation & Physicists' notation \\
\hline$\xi$ & $g$ \\
$R$ & $\mu R$ \\
$L$ & $\mu^{-1}$ \\
$K_{0}$ & $(R \Lambda)^{33 / 3}$ \\
$R L$ & $R$ \\
$K_{0}(R L)^{-1}$ & $\Lambda$ \\
\hline
\end{tabular}

The physicists then hold $R L / K_{0}$ constant while sending $L$ to zero (hence sending $R / K_{0}$ to infinity), again changing $\xi$ simultaneously so that the same combination of $R$ and $\xi$ remains fixed. (Here we are using our notation.) The only difference is that $K_{0}$ is not regarded as a constant, but rather as a parameter that tends to infinity as $R$ does.

(2) Equation (7.13) is related to the "renormalization group equation" as follows. When we vary parameters, the expectations $\langle\Phi\rangle$ become functions $\langle\Phi\rangle(R, \xi)$. In the physics literature it is assumed that the renormalized and unrenormalized expectations of Green functions $\Phi$ are related, to all orders in perturbation theory, by

$$
\langle\Phi\rangle^{\mathrm{re}}=Z(R)^{-d}\langle\Phi\rangle
$$

for some function $Z(R)$. With the requirement that $\langle\Phi\rangle^{\text {re }}$ be independent of $\xi$ and $R,(7.14)$ determines a relation between $\xi$ and $R$. Differentiating (7.14) with respect to $R$ then gives

$$
0=\left[R \frac{\partial}{\partial R}+\beta \frac{\partial}{\partial \xi^{2}}-d \gamma\right]\langle\Phi\rangle,
$$

where $\beta=R \frac{d \xi^{2}}{d R}$ and $\gamma=R Z^{-1} \frac{d Z}{d R}$. In general, $\beta$ has an expansion

$$
\beta=\beta_{1} \xi^{4}+\beta_{2} \xi^{6}+\ldots
$$

whose first coefficient can be computed using the semiclassical approximation (the higher terms involve perturbation theory beyond the semiclassical level). In fact, by (7.13) we have 


$$
\beta_{1}=-\frac{11}{12 \pi^{2}}
$$

This factor appears prominently in the physics literature whenever scaling behavior is discussed, although it is computed by entirely different methods. (It should be noted that the expression on the right-hand side of (7.13) differs from the $\beta$-function of the physicists at order $\xi^{6}$.) The fact that $\beta<0$ is interpreted to mean that Yang-Mills theory is asymptotically free (see [C], Chap. 5, Sect. 6, and Chap. 7, Sect. 3.6).

\section{Appendix}

This appendix contains the proof of several technical facts about $\zeta$-functions that were used in Sect. 3. These ensures that certain formal statements about $\zeta(s)$ are valid for large $s$, and remain valid for the analytic continuation. The proofs are based on heat kernel estimates.

First, suppose $D: \Gamma(E) \rightarrow \Gamma(E)$ is an operator as in the second paragraph of Sect. 3. Let $K_{D}$ be its heat kernel and set $k^{+}(t)=\int_{M} \operatorname{tr} \imath^{*} K_{D}^{+}(t)$.

Lemma A.1. (a) $I(s)=\int_{1}^{\infty} t^{s-1} k^{+}(t) d t$ is an entire function of $s$.

(b) If $\phi(t)=O\left(t^{L}\right)$ as $t \rightarrow 0$ then $\int_{0}^{1} t^{s-1} \phi(t) d t$ is holomorphic on the half-plane $\operatorname{Re}(s)>-L$.

Proof. Since the sum $\sum_{i} e^{-\lambda_{i} t}$ converges to $k^{+}(t)$ we have, for all $t>0$,

$$
k^{+}(t)=\sum_{i} e^{-\lambda_{i} t / 2} e^{-\lambda_{i} t / 2} \leqq e^{-\lambda_{1} t / 2} k^{+}(t / 2) \leqq e^{-\lambda_{1} t / 2} k^{+}(t / 2),
$$

where $\lambda_{1}>0$ is the first non-zero eigenvalue of $D$. It follows that the improper integral $I(s)$ is uniformly absolutely convergent, locally uniformly in $s$, for every $s \in \mathbf{C}$. Hence it represents an entire function of $s$. Similarly, the integral in (b) converges absolutely, locally uniformly in $s$, for $\operatorname{Re}(s)>-L$, and hence is holomorphic in that region.

Lemma A.2. Let $D$ be as above, and let $T: \Gamma(E) \rightarrow \Gamma(F)$ and $\widetilde{T}: \Gamma(F) \rightarrow \Gamma(E)$ be linear differential operators of order $\leqq k$. Then $T e^{-t D} \widetilde{T}, e^{-t D} \widetilde{T} T$, and $\widetilde{T} T e^{-t D}$ are all traceclass, and

$$
\operatorname{Tr}\left(T e^{-t D} \widetilde{T}\right)=\operatorname{Tr}\left(e^{-t D} \tilde{T} T\right)=\operatorname{Tr}\left(\tilde{T} T e^{-t D}\right) .
$$

Proof. Extend $T, \widetilde{T}$, and $e^{-t D}$ by zero to endomorphisms of $\Gamma(E) \oplus \Gamma(F)$. Write

$$
T e^{-t D} \widetilde{T}=\left(T e^{-\frac{t}{2} D}\right)\left(e^{-\frac{t}{2} D} \tilde{T}\right) \text { and } T e^{-\frac{t}{2} D}=\left(T e^{-\frac{t}{4} D}\right)\left(e^{-\frac{t}{4} D}\right) \text {. }
$$

Since $T: L_{k}^{2}(E \oplus F) \rightarrow L^{2}(E \oplus F)$ and

are bounded, so is

$$
e^{-\frac{t}{4} D}: L^{2} \rightarrow L_{k}^{2}
$$

$$
T e^{-\frac{t}{2} D}: L_{k}^{2} \rightarrow L_{k}^{2} .
$$


Since $e^{-\frac{t}{4} D}$ is trace-class, so is its product with the bounded operator $T e^{-\frac{t}{4} D}$, so $T e^{-\frac{t}{2} D}$ is trace-class. The same applies for $e^{-\frac{t}{2} D} \tilde{T}$, and hence the product $T e^{-t D} \widetilde{T}$ is trace-class. Since $\operatorname{Tr}(A B)=\operatorname{Tr}(B A)$ for trace-class operators $A, B$, we have

$$
\operatorname{Tr}\left(T e^{-t D} \widetilde{T}\right)=\operatorname{Tr}\left(e^{-\frac{t}{2} D} \widetilde{T} T e^{-\frac{t}{2} D}\right)
$$

Similar arguments show that $\widetilde{T} T e^{-\frac{t}{2} D}$ is bounded from $L^{2}$ to $L^{2}$, and $e^{-\frac{t}{2} D}$ is traceclass, so we may reverse the order of these two factors in the trace, obtaining $\operatorname{Tr}\left(\widetilde{T} T e^{-t D}\right)$. The second equality in (A.1) follows similarly.

Lemma A.3. Let $f$ be a continuous function on $M$, and let $D$ be as above. Then for $t>0, f e^{-t D}$ and $f D e^{-t D}$ are trace-class, and

$$
\begin{gathered}
\operatorname{Tr}\left(f D e^{-t D}\right)=-\frac{d}{d t} \operatorname{Tr}\left(f e^{-t D}\right), \\
\operatorname{Tr}\left(f e^{-t D}\right)=\int_{M} f \operatorname{tr} \imath^{*} K_{D}(t) .
\end{gathered}
$$

Proof. The previous lemma implies that $f e^{-t D}$ and $f D e^{-t D}$ are trace-class. Let $\left\{\phi_{j}\right\}$ be an orthonormal eigenbasis of $L^{2}$ with respect to $D$. The final summation in the bound

$$
\sum_{j}\left|\left\langle f D e^{-t D} \phi_{j}, \phi_{j}\right\rangle\right|=\sum_{j}\left|\left\langle f \lambda_{j} e^{-t \lambda_{j}} \phi_{j}, \phi_{j}\right\rangle\right| \leqq\|f\|_{\infty} \sum_{j} \lambda_{j} e^{-t \lambda_{j}}
$$

converges absolutely and uniformly for $t \geqq \varepsilon>0$ to $\int_{M} \partial_{t} \iota^{*} K_{D}(t)$, so the sum

$$
\operatorname{Tr}\left(f D e^{-t D}\right)=\sum_{j}\left\langle f D e^{-t D} \phi_{j}, \phi_{j}\right\rangle
$$

converges locally uniformly in $t$. Since this sum is the term-by-term derivative of $-\sum e^{-t \lambda_{j}}\left\langle f \phi_{j}, \phi_{j}\right\rangle=-\operatorname{Tr}\left(f e^{-t D}\right)$, statement (a) follows. For (b), we have

$$
\operatorname{Tr}\left(f e^{-t D}\right)=\sum_{j} e^{-t \lambda_{j}} \int_{M} f\left|\phi_{j}\right|^{2}=\int_{M} f \sum_{j} e^{-t \lambda_{j}}\left|\phi_{j}\right|^{2} \int_{M} f(x) \operatorname{tr} \imath^{*} K_{D}(t) .
$$

In the remaining lemmas $D_{\alpha}$ will be one of the families $\widetilde{\Delta}_{A_{\alpha_{\nu}}}^{i}$ or $\tilde{\triangle}_{A_{\alpha}}^{-}$, where $\alpha \in \mathbf{R}$ and $v \in \mathbf{R}^{5}$. As in (3.17) we let $B_{\alpha}(s)=\Gamma(s) \zeta_{D_{\alpha}}(s)$ and write $\delta$ for $\frac{d}{d \alpha}$. Note that $\operatorname{dim} \operatorname{ker} D_{\alpha}$ is constant for these families.

Lemma A.4. Let $\left(\delta D_{\alpha}\right)_{z}$ denote the action of $\delta D$ on the $z$-variable. Then

$$
\delta K_{\alpha}(t, x, y)=-\int_{0}^{t} d \tau\left[\int_{M} K_{\alpha}(t-\tau, x, z)\left(\delta D_{\alpha}\right)_{z} K_{\alpha}(\tau, z, y)\right] .
$$

Proof. Fix $\varepsilon>0$ and consider the integral

$$
I_{\varepsilon}=-\int_{\varepsilon}^{t-\varepsilon} d \tau\left[\int_{M} K_{\alpha}(t-\tau, x, z)\left(\delta D_{\alpha} K_{\alpha}\right)(\tau, z, y)\right] .
$$

Differentiating (3.3) with respect to $\alpha$ and substituting gives

$$
I_{\varepsilon}=\int_{\varepsilon}^{t-\varepsilon} d \tau\left[\int_{M} K_{\alpha}(t-\tau, x, z)\left(\left(\partial_{\tau}+D_{\alpha}\right)\left(\delta K_{\alpha}\right)(\tau, z, y)\right] .\right.
$$


If we regard the heat kernel as a function (rather than a density) we can rewrite the term involving $D_{\alpha} \delta K_{\alpha}$ as

$$
\int_{\varepsilon}^{t-\varepsilon} d \tau\left[\int_{M} K_{\alpha}(t-\tau, x, z)\left(D_{\alpha}\right)_{z}\left(\delta K_{\alpha}\right)(\tau, z, y) d z\right] .
$$

Now use self-adjointness of $D_{\alpha}$ and the fact that $K_{\alpha}$ satisfies the heat equation (3.1). Replacing $\partial_{\tau} K_{\alpha}(t-\tau, \cdot, \cdot)$ with $-\partial_{\tau} K_{\alpha}(t-\tau, \cdot, \cdot)$ the above then becomes

$$
\int_{\varepsilon}^{t-\varepsilon} d \tau\left[\int_{M}\left(\partial_{t} K_{\alpha}\right)(t-\tau, x, z)\left(\delta K_{\alpha}\right)(\tau, z, y) d z\right] .
$$

Hence $I_{\varepsilon}$ is a total time derivative, which simplifies to

$$
I_{\varepsilon}=\int_{M} K_{\alpha}(\varepsilon, x, z) \delta K_{\alpha}(t-\varepsilon, z, y) d z-\int_{M} K_{\alpha}(t-\varepsilon, x, z) \delta K_{\alpha}(\varepsilon, z, y) d z .
$$

As $\varepsilon \rightarrow 0$, the first of these integrals approaches $\delta K_{\alpha}(t, x, y)$. Furthermore, (i) $K_{\alpha}(t-\varepsilon, x, z)$ is bounded, locally uniformly in $\alpha$, as $\varepsilon \rightarrow 0$, and (ii) since each $K_{\alpha}$ has the same leading term in its parametrix (3.5) we have $\delta K_{\alpha}(\varepsilon, z, y) \rightarrow 0$ when $\operatorname{dist}(z, y)>\delta_{0}$ and

$$
\left|\delta K_{\alpha}(\varepsilon, x, y)\right| \leqq C \varepsilon^{1-\frac{n}{2}} \exp \left(\frac{-c}{\varepsilon} d^{2}(z, y)\right)
$$

when $\operatorname{dist}(z, y) \leqq \delta_{0}$. It follows that the second integral above vanishes as $\varepsilon \rightarrow 0$.

Lemma A.5. For $t>0,\left(\delta D_{\alpha}\right) e^{-t D_{\alpha}}$ is trace-class, and if $\operatorname{dim} \operatorname{ker} D_{\alpha}$ is independent of $\alpha$, then for $\operatorname{Re}(s) \gg 0$,

$$
\delta B_{\alpha}(s)=-\int_{0}^{\infty} t^{s}\left(\operatorname{Tr}\left(\delta D_{\alpha}\right) e^{-t D_{\alpha}}\right) d t .
$$

Proof. The proof of Lemma A.2 shows that $\left(\delta D_{\alpha}\right) e^{-\tau D_{\alpha}}$ is trace-class. To obtain a specific bound, write $\operatorname{Tr}^{0}=\operatorname{Tr}_{\alpha}^{0}$ for the trace on the subspace $\operatorname{ker}\left(D_{\alpha}\right), \operatorname{Tr}^{+}$for the trace on the positive eigenspace, and let $\pi=\pi_{\alpha}$ be the orthogonal projection onto $\operatorname{ker}\left(D_{\alpha}\right)$. Then $\pi \circ D_{\alpha}=D_{\alpha} \circ \pi=0$, and therefore

$$
\operatorname{Tr}^{0}\left(\delta D e^{-t D}\right)=\operatorname{Tr}(\pi \circ \delta D \circ \pi)=-\operatorname{Tr}(\pi \circ D \circ \delta \pi)=0
$$

for each $\alpha$. Consequently,

$$
\operatorname{Tr}\left(\delta D_{\alpha} e^{-t D_{\alpha}}\right)=\operatorname{Tr}^{+}\left(\left(\delta D_{\alpha} e^{-t / 2 D_{\alpha}}\right) e^{-t / 2 D_{\alpha}}\right) \leqq C e^{-t \lambda_{1}(\alpha) / 2},
$$

where $\dot{\lambda}_{1}(\alpha)$ is the first positive eigenvalue of $D_{\alpha}$. Moreover, since $\lambda_{1}$ depends continuously on $\alpha$ this exponential decay rate is locally uniform in $\alpha$. The existence of a parametrix for the heat kernel implies that $\operatorname{Tr}\left(\delta D_{\alpha} e^{-t D_{\alpha}}\right)=O\left(t^{-N}\right)$ for some $N$ as $t \rightarrow 0$. Hence for $\operatorname{Re}(s)$ sufficiently large, the integral on the right-hand side of (A.2) converges absolutely, locally uniformly in $s$ and $\alpha$.

Now, if we set $y=x$ in Lemma A.4 and integrate over $M$, the integrals converge uniformly in $\alpha$. Hence $\int_{M} \delta l^{*} K_{D_{\alpha}}(t)=\delta \int_{M} \imath^{*} K_{D_{\alpha}}(t)$. Applying Lemmas A.3b and A.2, we therefore obtain

$$
\delta\left(\operatorname{Tr}\left(e^{-t D}\right)\right)=-\int_{0}^{t} \operatorname{Tr}\left(e^{-(t-\tau) D} \delta D e^{-\tau D}\right) d \tau=-\int_{0}^{t} \operatorname{Tr}\left(\delta D e^{-\tau D} e^{-(t-\tau) D}\right) d \tau,
$$


where $e^{-\tau D} e^{-(t-\tau) D}=e^{-t D}$ is independent of $\tau$. Since

$$
\operatorname{Tr}\left(e^{-t D}\right)=\operatorname{Tr}^{+}\left(e^{-t D}\right)+\operatorname{dim} \operatorname{ker} D
$$

and $\operatorname{dim} \operatorname{ker} D_{\alpha}$ is constant this reduces to

$$
\delta\left(\operatorname{Tr}^{+}\left(e^{-t D}\right)\right)=\delta\left(\operatorname{Tr}\left(e^{-t D}\right)\right)=-t \operatorname{Tr}\left(\delta D_{\alpha} e^{-t D_{\alpha}}\right) .
$$

From the definition of $B_{\alpha}$ and the integral expression (3.9) for the $\zeta$-function we have

$$
\delta B_{\alpha}(s)=\delta \int_{0}^{\infty} t^{s-1} \operatorname{Tr}^{+}\left(e^{-t D_{\alpha}}\right) d t .
$$

But we now know that $t^{s-1} \operatorname{Tr}^{+}\left(e^{-t D}\right)$ and $t^{s-1} \delta\left(\operatorname{Tr}^{+}\left(e^{-t D}\right)\right)=-t^{s}\left(\operatorname{Tr} \delta\left(e^{-t D}\right)\right)$ are differentiable as functions of $\alpha$ and integrable as functions of $t$, locally uniformly in $\alpha$. Hence we can move the $\delta$ inside the integral and use (A.4) to obtain (A.2).

Lemma A.6. Write $B_{\alpha}(s)=\zeta_{D_{\alpha}}(0)\left(\frac{1}{s}-\gamma\right)+\zeta_{D_{\alpha}}^{\prime}(0)+O(s)$ as in (3.17). Then $\delta\left(\zeta_{D_{\alpha}}^{\prime}(0)\right)=\left(\delta \zeta_{D_{\alpha}}\right)^{\prime}(0)$, and near $s=0$,

$$
(\delta B)(s)=\delta\left(\zeta_{D}(0)\right)\left(\frac{1}{s}-\gamma\right)+\delta\left(\zeta_{D}^{\prime}(0)\right)+O(s) .
$$

Proof. The arguments used in the proof of Lemma A.5 show that for $\operatorname{Re}(s) \gg 0$ the integral

$$
-\frac{d}{d s} \int_{0}^{\infty} t^{s}\left(\operatorname{Tr}\left(\delta D_{\alpha}\right) e^{-t D_{\alpha}}\right) d t=-\int_{0}^{\infty} t^{s} \log t\left(\operatorname{Tr}\left(\delta D_{\alpha}\right) e^{-t D_{\alpha}}\right) d t
$$

is absolutely convergent, locally uniformly in $s$. By (A.2) it is equal to $\frac{d}{d s}\left(\delta B_{\alpha}\right)(s)$. On the other hand, using (A.4) and differentiating under the integral sign as in (A.5), it is also equal to

$$
\delta \int_{0}^{\infty} t^{s-1} \log t \operatorname{Tr}\left(e^{-t D}\right) d t=\delta \int_{0}^{\infty} \frac{d}{d s}\left(t^{s-1} \operatorname{Tr}\left(e^{-t D}\right)\right) d t .
$$

This last integral is also locally uniformly absolutely convergent in $s$, so we can pull the $\frac{d}{d s}$ outside the integral. We conclude that

$$
\delta \frac{d}{d s} B_{\alpha}(s)=\frac{d}{d s} \delta B_{\alpha}(s)
$$

for $\operatorname{Re}(s) \gg 0$. In particular, $\frac{d}{d s} \delta B_{\alpha}$ exists, so $\delta B_{\alpha}$ is analytic in this region.

To describe the analytic continuation of $\delta A_{\alpha}$ to $\mathbf{C}$, we write (A.2) as

$$
-\delta B_{\alpha}(s)=\int_{0}^{1} t^{s} \operatorname{Tr}\left(\delta D e^{-t D}\right) d t+\int_{1}^{\infty} t^{s} \operatorname{Tr}\left(\delta D e^{-t D}\right) d t .
$$

As in the proof of Lemma A.1, the exponential decay (A.4) implies that the second of these integrals is an entire function. We can rewrite the first integral by 
substituting in (A.4), using Lemma A.3b, and then substituting in the parametrix (3.5); it becomes

$$
-\int_{0}^{1} t^{s-1}\left[\sum_{k=0}^{L}\left(\delta \hat{\alpha}_{k}\right) t^{k-\frac{n}{2}}+\phi(t)\right] d t
$$

where $\hat{a}_{k}=(4 \pi)^{-\frac{\pi}{2}} \int_{M} \operatorname{tr} l^{*} a_{k}$ and $\varphi(t)=O\left(t^{L-\frac{n}{2}}\right)$. By Lemma A.1 the $\phi$ integral is holomorphic for $\operatorname{Re}(s) \gg 0$. Integrating the terms in the summation, we find that

$$
-\delta \mathrm{B}_{\alpha}=\left(\sum_{k=0}^{L} \frac{\delta \hat{a}_{k}}{s+k-n / 2}\right)+\Phi(s),
$$

where $\Phi(s)$ is holomorphic in the half-plane $H_{L}=\left\{s \mid \operatorname{Re}(s)>\frac{n}{2}-1-L\right\}$. Thus the right-hand side is equal to the analytic continuation of $-\delta B_{\alpha}$ on $H_{L}$. Furthermore, the arguments of the first paragraph of this proof apply to the two integrals that comprise the $\Phi$ term above, so (A.7) holds on $H_{L}$. Since $L$ is arbitrary, we see that $-\delta B_{\alpha}$ extends meromorphically to $C$ with only simple poles, and that the locations of the poles form a subset of the set of integers $P=\left\{\frac{n}{2}-1, \frac{n}{2}-2, \frac{n}{2}-3, \ldots\right\}$. This set $P$ is independent of $\alpha$ and (A.7) holds for all $s \notin P$.

The same derivation works when $B_{\alpha}(s)$ is replaced by $s B_{\alpha}(s)$ or by $B_{\alpha}(s) / \Gamma(s)$ $=\zeta_{D_{\alpha}}(s)$, which have no pole at $s=0$. In particular, $\delta \zeta_{D_{\alpha}}$ is analytic,

and

$$
\delta\left(\zeta_{D_{\alpha}}^{\prime}(0)\right)=\left(\delta \zeta_{D_{\alpha}}\right)^{\prime}(0)
$$

$$
\begin{aligned}
(\delta B)(s)=\Gamma(s)\left(\delta \zeta_{D}\right)(s) & =\left(\frac{1}{s}-\gamma+O(s)\right)\left(\delta \zeta_{D}(0)+s\left(\delta \zeta_{D}\right)^{\prime}(0)+O(s)\right) \\
& =\left(\frac{1}{s}-\gamma\right)\left(\delta \zeta_{D}(0)+\delta\left(\zeta_{D}^{\prime}\right)(0)\right)+O(s) .
\end{aligned}
$$

\section{References}

[AHDM] Atiyah, M.F., Hitchin, N.J., Drinfeld, V.G., Manin, Yu.I.: Construction of instantons. Phys. Lett. 65A, 185-187 (1978)

[AHS] Atiyah, M.F., Hitchin, N.J., Singer, I.M.: Self-duality in four dimensional Riemannian geometry. Proc. R. Soc. Lond. Ser. A 362, 425-461 (1978)

[BP] Belavin, A.A., Polyakov, A.M.: Quantum fluctuations of pseudoparticles. Nucl. Phys. B 123, 429-444 (1977)

[BV] Babelon, O., Viallet, C.M.: The geometrical interpretation of the Faddeev-Popov determinant. Phys. Lett. 85B, 246-248 (1979)

[C] Coleman, S.: Aspects of Symmetry, Chap. 5: Secret Symmetry: An introduction to spontaneous symmetry breakdown and gauge fields, and Chap. 7: The Uses of Instantons. Cambridge: Cambridge University 1985

[CVDN] Chadha, S., di Vecchia, P., D'Adda, A., Nicodemi, F.: $\zeta$-function regularization of the quantum fluctuations around the Yang-Mills pseudoparticle. Phys. Lett. 72B, 103-108 (1977)

[DMM] Doi, H., Matsumoto, Y., Matumoto, T.: An explicit formula of the metric on the moduli space of BPST-instantons over $S^{4}$. A Fete of Topology, pp. 543-556. New York: Academic Press 1988 
[FU] Freed, D.S., Uhlenbeck, K.K.: Instantons and Four-Manifolds. Berlin, Heidelberg, New York: Springer 1984

[F] Friedman, A.: Partial differential equations of parabolic type. Englewood Cliffs, NJ: Prentice-Hall 1971

[G] Gilkey, P.: The spectral geometry of a Riemannian manifold. J. Differ. Geom. 10, 601-618 (1973)

[GP 1] Groisser, D., Parker, T.H.: The Riemannian geometry of the Yang-Mills moduli space. Commun. Math. Phys. 112, 663-689 (1987)

[GP 2] Groisser, D., Parker, T.H.: The geometry of the Yang-Mills moduli space for definite manifolds. J. Differ. Geom. 29, 499-544 (1989)

[Gr] Groisser, D.: The geometry of the moduli space of $\mathbf{C P}^{2}$ instantons. Invent. Math. 99, 393-409 (1990)

[H] 't Hooft, G.: Computation of the quantum efects due to a four-dimensional pseudoparticle. Phys. Rev. D 14, 3432-3450 (1976)

[L] Lawson, H.B.: The theory of gauge fields in four dimensions. Providence, RI: Am. Math. Soc. 1986

[MSZ] Montgomery, D., Samelson, H., Zippin, L.: Exceptional orbits of highest dimension. Ann. Math. 64, 131-141 (1956)

[O] Osborn, H.: Semiclassical functional integrals for self-dual gauge fields. Ann. Phys. 135, 373-415 (1981)

[P] Patodi, V.K.: Curvature and the eigenvalues of the Laplace operator. J. Differ. Geom. 5, 233-249 (1971)

[PR] Parker, T.H., Rosenberg, S.: Invariants of conformal Laplacians. J. Differ. Geom. 25, 199-222 (1987)

[RaSi] . Ray, D.B., Singer, I.M.: R-torsion and the Laplacian on Riemannian manifolds. Adv. Math. 7, 145-210 (1971)

[RoSc] Romanov, V.N., Schwarz, A.S.: Anomalies and elliptic operators. Theor. Math. Phys. (English Translation) 41, 967-977 (1979)

[S] Seeley, R.: Complex powers of an elliptic operator. Proc. Symp. Pure Math. 10, 288-307 (1973)

[Sc] Schwarz, A.S.: Instantons and fermions in the field of instanton. Commun. Math. Phys. 64, 233-268 (1979)

Communicated by A. Jaffe 